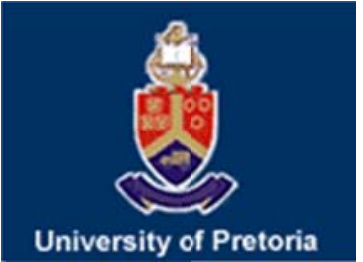

University of Pretoria Department of Economics Working Paper Series

\title{
Conflict Heterogeneity in Africa
}

Carolyn Chisadza

University of Pretoria

Matthew Clance

University of Pretoria

Working Paper: 2018-52

August 2018

Department of Economics

University of Pretoria

0002, Pretoria

South Africa

Tel: +27 124202413 


\title{
Conflict Heterogeneity in Africa*
}

\author{
Carolyn Chisadza ${ }^{\dagger} \quad$ Matthew Clance C $^{\ddagger}$
}

August 17, 2018

\begin{abstract}
One of the Sustainable Development Goals is to significantly reduce all forms of violence and related death rates everywhere. The economic development literature widely concurs that conflicts have adverse economic consequences that contribute to poverty, disinvestment, and lower human capital leading to widespread inequality and lower economic growth. As such, understanding the nature of conflict has been an important focus for political leaders, policymakers and researchers alike. However, the existing literature does not typically distinguish between the types of conflict, and empirical evidence relies on the assumption that effects are similar across the world. Using panel data analysis and a comprehensive disaggregated georeferenced conflict dataset from 1997 to 2016, we find significant evidence of heterogeneity in the predictors' effects on different types of conflicts in Africa.
\end{abstract}

Keywords: conflict, regional heterogeneity, panel data

JEL Classification: C33, H56, O10, O43

${ }^{*}$ We acknowledge comments received at the Economic Society of South Africa (ESSA) Conference in Grahamstown, and the brown bag seminars (University of Pretoria).

${ }^{\dagger}$ Department of Economics, University of Pretoria, Private Bag X20, Hatfield 0028, South Africa, E-mail: carolyn.chisadza@up.ac.za.

${ }^{\ddagger}$ Department of Economics, University of Pretoria, Private Bag X20, Hatfield 0028, South Africa, E-mail: matthew.clance@up.ac.za. 


\section{Introduction}

The literature widely concurs that conflicts have adverse economic consequences that persist for many years even spreading beyond a country's borders. Conflicts not only contribute to poverty, disinvestment and disease outbreaks, but also increase widespread inequality and lower human capital delaying key 2030 targets of the Sustainable Development Goals. ${ }^{1}$ In turn, lower economic development carries the risk of an increase in conflicts and countries often find themselves caught in the conflict trap. Analyzing how conflicts respond to economic and social determinants has been the focus of much research. However the previous analyses have mostly treated incidences of conflicts as homogeneous outcomes without distinguishable differences across the world (Collier and Hoeffler (1998), Collier and Hoeffler (2004a), Fearon and Laitin (2003), Hegre and Sambanis (2006), Collier et al. (2009)). The existing literature also does not typically identify the actors contributing to conflict as a source of analysis, preferring broad terms, such as civil or ethnic (Blattman and Miguel (2010).

We argue that the treatment of conflict effects as homogeneous across regions and types of aggressors result in misleading conclusions in previous studies of conflict. Social and economic circumstances can differ across regions while governments and groups ${ }^{2}$ can have different support from the surrounding regions to provide their policies. For the purpose of this study, we focus on Africa because it is considered a high conflict area with repeated episodes occurring despite attempts by governments and international organisations ${ }^{3}$ to combat conflict (Collier and Hoeffler (2002), Miguel et al. (2004), Arezki and Gylfason (2013)). We also expect more variation in the predictors as the continent is still developing economically and politically.

Figure 1 indicates the intensity of conflicts across Africa for each country for the period 1997 to 2017. To make intensity measures comparable during the period, we divide the number of total conflicts over the period by country's area in square kilometres and group countries into two quantiles. The darker shaded polygons indicate a higher intensity of conflicts in countries over the period relative to their geographic size. Figure 1 suggests evidence of significant episodes of conflicts occurring around the Sahel region, the horn of Africa and the south of Africa but identification of the types of conflicts is not discernible. This oversight in most of the literature guides the basis of our study: to identify the countries in Africa that are most affected by conflicts, the type of conflict dominating these countries and the likely determinants contributing to that type of conflict.

First, we focus on the actors of a particular "event" during the period 1997 to 2017 by disaggregating conflict into state-based (government and related entities), non state-based (militias/ethnic/rebel

\footnotetext{
${ }^{1}$ The rate of children leaving primary school in conflict affected countries reached $50 \%$ in 2011 , which accounts for 28.5 million children, showing the impact of unstable societies on one of the major goals of the post 2015 agenda: education (http://www.un.org/sustainabledevelopment/peace-justice/)

${ }^{2}$ Groups or communities have taken it upon themselves to fill the roles of services not provided by the government whether for the good or bad of society. Citizens turn to vigilantes as they try to protect their neighbourhoods from gang wars and drugs in the Eastern Cape of South Africa, or villagers try to protect their daughters from Boko Haram in Nigeria.

${ }^{3}$ These include the United Nations and African Union peacekeeping operations.
} 
groups), and civilians (protesters/rioters). ${ }^{4}$ Second, we group the countries according to the intensity of each type of conflict over the period of analysis. The disaggregation allows for identification of the type of conflict contributing to overall conflict on the continent and to distinguish differences across particular instigators of conflict. To illustrate, Figure 2 indicates that the main contributor to overall conflict in Africa between 1997 and 2017 was non state-based conflict given the number of events and countries with high intensity grouped above and below the eastern Sahel region compared to state-based and civilian-based conflicts. Figure 2 also indicates that countries with high intensity of all conflict types include Egypt, Ethiopia, Kenya, Nigeria, Somalia, Sudan, Tunisia, Uganda and Zimbabwe while Angola and Equatorial Guinea are influenced most by state-based conflicts. Similarly, Cameroon and the Central African Republic are most affected by non statebased conflicts and Burkina Faso, Ghana and Togo suffer most from civilian based conflicts. These types of distinctions can at least serve as a starting basis for policy targets.

In pursuing this research we hope that the results will bring awareness to the nuances in assessing conflict. As such, policy recommendations should be carefully considered given the type of conflict in each country if we want to come close to meeting the 2030 targets of reduced violence and promoting sustained economic growth across the world.

\section{Data and Methodology}

The conflict data is from the Armed Conflict Location and Event Data project (or ACLED) and we use the data specific to Africa during the period 1997-2015 (Raleigh et al. (2010)). ACLED is georeferenced data that we aggregate to a count of the number of "events" in a country in a year and disaggregate the events by aggressor type: 1) recognized Governments or related parties, 2) Militias and Rebels, and 3) civilian action. ${ }^{5}$ Conflict is defined as an event when an aggressor uses lethal force against another group or civilians. Intensity groupings are created using the number of each type of event over the entire period and dividing by the country's geographic size. The intensity groupings are used to divide the sample and allow for heterogeneity across intensity groupings. ${ }^{6}$

Determinants of conflict have traditionally been estimated using Ordinary Least Squares (OLS), binary limited dependent variable models such as a probit or logit (Collier and Hoeffler (1998), Collier and Hoeffler (2004a), Fearon and Laitin (2003)), or proportional hazard model to analyse conflict duration (Collier et al. (2004), Rouen and Sobek (2004)). Many researchers model the frequency of events, such as conflicts and wars, as continuous processes using OLS. Our aggregation

\footnotetext{
${ }^{4}$ We also have a fourth group on 'external' forces, but the observations are few for Africa so we drop this group from our analysis.

${ }^{5}$ Governments include related parties such as military, police forces, and security forces. Militias include groups that identify or organize with a particular political, community, ethnic, or religious ideology. Civilian action includes rioters and protesters.

${ }^{6}$ We use two groupings but results for three and four groupings are available upon request. For example, the lowest quantile in the state-based conflicts would represent countries with $0-50 \%$ occurrences of conflict, while the highest quantile would represent countries with 51-100\% occurrences. A list of countries by type of conflict and quantiles can be found in Table 1 of Appendix A.
} 
of events for each country in a given year means that count models are possibly more appropriate and our choice is the negative binomial. ${ }^{7}$ Wooldridge (2010) notes that OLS is not ideal since $E\left(V_{i j t} \mid X\right)$ can be negative even when $V_{i j t}$ is non-negative, where $V_{i j t}$ is the count of the number of events. The model is defined as

$$
E\left[V_{i j t} \mid x_{i t}, \phi_{i}, \epsilon_{i t j}\right]=\exp \left(\gamma+\beta x_{i t}+\epsilon_{i t j}\right)
$$

where $V_{i j t}$ is the count of events per land size area for country $i$, conflict type $j$, and year $t, x_{i t}$ is a vector of determinants of conflict described next, and $\epsilon_{i t j}$ is unobserved heterogeneity. ${ }^{8}$

We compare economic, social, and political determinants to analyse unique effects across the aggregate and disaggregation of events, and the intensity groupings. The economic variables include income per capita at 2005 constant prices, military expenditure as a percentage of GDP, and natural resource rents as a percentage of GDP that are obtained from the World Development Indicators (WDIs). We also include a globalisation index for openness compiled by (Dreher, 2006) and updated by (Dreher et al., 2008). The globalisation index combines three key components of globalisation (political, economic and social globalisation) into a weighted index ranging from 0 (no globalisation) to 100 (highly globalised). The index captures international flows of goods, capital, businesses, people, technology, information and the presence of international organisations.

The social variables include gross primary enrollment rates and population density measured as people per square kilometre of land area from the WDI. The political variable, from the Center for Systemic Peace, is the state fragility index which scores each country on both effectiveness and legitimacy in four performance dimensions: security, political, economic and social. It measures a country's ability to fulfill basic functions, such as manage conflict, implement public policy, deliver public services and sustain progressive development. The index ranges from 0 (no fragility) to 25 (extreme fragility).

\section{Results}

The study highlights the presence of heterogeneity in some determinants of conflict across types of conflicts and intensity groups. A more comprehensive study is required to determine why similar determinants affect conflict types differently across countries. For the current study, previous literature will guide the interpretation of the results. The results section is structured with Table 1 displaying "All Conflicts" and "Government" and Table 2 with "Groups/Militias" and "Civilians/Protests" across quantiles. We also include squared terms to observe possible non-linearities in the determinants. The results of the determinants with similar effects are presented in Section

\footnotetext{
${ }^{7}$ We could use the estimate techniques previously mentioned but our preferred method is the count model negative binomial given that the dependent variable is a discrete nonnegative integer value. Results using OLS, probit, and logit are available upon request. The negative binomial was chosen over the Poisson because there were signs of overdispersion.

${ }^{8}$ See Cameron and Trivedi (2005) for the the specific functional form of the conditional log likelihood.
} 
3.1 and others are presented in Section 3.2. Tables 3 to 6 are robustness results and discussed in Section 4.

\subsection{Homogeneous Effects}

Globalisation is positive and significant across aggregate and disaggregate conflict types. This result is consistent with studies that find globalisation increases conflict by creating conditions that increase income inequality and poverty, as well as facilitating social breakdown because of resistance from the oppressed ((Olzak, 2011), (Bezemer and Jong-A-Pin, 2013)). Our initial results are in contrast to previous evidence showing globalisation has a pacifying effect by promoting economic and social growth through trade, information and technology transfers, and the migration of people. These factors encourage peaceful relationships amongst countries and decrease both the likelihood and severity of conflict ((Blanton and Apodaca, 2007), (Choi, 2010), (Flaten and de Soysa, 2012), (Hegre et al., 2010)). The implied globalisation effect on conflict of a one percentage change is relatively large, corresponding to an increase of 2 to 5 percentage increase in conflict. Civilian based violence is consistently on the larger side of this increase. Globalisation facilitates the transfer of high and low skilled labour that can lead to resentment from locals who are competing for similar jobs. ${ }^{9}$. Other reasons include unfavourable trade balance that crowd out local producers causing social unrest or higher growth increasing economic competition between countries for investments. In section 4, we further disaggregate the components of globalisation to show that political openness is causing this positive effect on conflict and other forms of openness, such as economic, are decreasing conflict.

The inclusion of the squared term for globalisation reveals a "u" shaped non-linear relationship with conflict. Initial levels of globalisation are associated with decreased conflicts, but this changes over time with increased globalisation. This non-linearity holds across the different conflict types. The initial negative effects on conflict may occur at the onset of peace treaties between countries generating a decrease in militarised interstate disputes (Choi, 2010). However, in sub-Saharan Africa, several of these ceasefires have failed to prevail, for example, the Lusaka Accords (1994) in Angola or the four peace agreements that were ineffective in curbing the violence in the DRC. ${ }^{10}$

Countries characterised by higher natural resource rents, military expenditure, and weak institutions have increased conflicts across all types and intensity groups. The African continent's vast resources have long been a source of contention among governments and individuals leading to conflict (Fearon and Laitin (2003), (Collier and Hoeffler (2004a), Barbieri and Reuveny (2005)). Resource rents provide motivation and opportunities for governments and disgruntled groups to support themselves through expropriation, particularly when it comes to control of state power. The effects of resource rents are mainly consistent for relatively high intense conflicts. We observe an inverted non-linear relationship between resource rents and conflict. Initial increases in resource rents only serve to

\footnotetext{
${ }^{9}$ An example of this includes the xenophobic attacks in South Africa

${ }^{10}$ The Lusaka Ceasefire Agreement (1999), the Sun City Agreement (2002), the Pretoria Agreement (2002) and the Luanda Agreement (2002)
} 
worsen all conflict types, however over time there is an expected change as resource rents continue to rise. This change may be through the leaders' increased opportunity costs of losing control over the resources if they engage in conflict.

According to Collier and Hoeffler (2004b), military spending can deter conflict by lowering the prospects of rebel success. In contrast, increased military spending post-conflict can increase the risk of renewed conflict as opponents could fear a breach of peace settlement. Interestingly, the nonlinear relationship captures both sides of the argument. For Africa, we find that initially, military spending reduces conflict, but over time continuous military spending appears to send the wrong signal across borders and within one's own borders.

Fragile states are institutionally weak and therefore more susceptible to conflict (Fearon and Laitin (2003)). According to Rouen and Sobek (2004), weak institutions can be ineffective in restraining rebel groups. Moreover, Olzak (2011) attributes strong state capacity to decreased conflict as strong states have the capacity to suppress civil wars, compared to weak states, through superior military and policing strength, and strong bureaucratic administrations. We do not observe non-linearity with state fragility.

\section{$3.2 \quad$ Hetergeneous Effects}

Heterogeneous effects are found in the determinants of income per capita, population density, and primary education. Collier and Hoeffler (2004a) finds that reductions in conflict are possible through increasing the standards of living and lowering the risk of conflict over grievances or incurring high opportunity costs from instability due to conflict. We find that income per capita increases high intensity conflicts for civilian-based violence and increases low intensity conflicts for state-based violence. Income per capita in Africa is still low relative to other continents. This can increase the likelihood of inequality resulting in civil unrest among the poor, including marginalised tribes or ethnic groups (Michalopoulos and Papaioannou (2016), Gleditsch (2007)). Collier and Hoeffler (2002) and Fearon and Laitin (2003) find that poverty facilitates recruitment of civilians for rebel groups as income opportunities worsen within formal labour market. Examples include Zimbabwe's worsening poverty conditions resulting in several civilian uprisings against the incumbent government and South Africa's increasing inequality causing more frequent protests by trade unions and poor.

Civilian violence follows an inverted non-linear relationship with income per capita. Initial low levels of income may increase the opportunity cost of engaging in conflict. Higher levels of income may increase inequality within a country. However, countries with low levels of income per capita are more likely to be involved in group-based conflicts, but this relationship is expected to change as the countries' levels of income per capita increases over time. As stated earlier, poverty can increase

grievances. The wealthier a country becomes, the more resources it has to sustain its population which decreases the risk of conflicts over grievances (Collier and Hoeffler (2004a)). A wealthier country can also afford a bigger police and military force to keep the peace (Pinker (2011)). 
The effects of population density are negative and significant for the low intensity levels within groups-based violence, but insignificant for other conflict types. Denser populations become more difficult to co-ordinate for rebel groups which may explain the negative relationship. Interestingly, the inclusion of the squared term indicates a similar inverted non-linear relationship across all three conflict types. Sparse populations are more prone to conflict because they inhibit government capability for protection, especially if they are spread outside urban areas, but they also become easier targets for rebel groups (Collier and Hoeffler (2004a)). Conflict reduces as the population becomes more concentrated. Our results are not consistent with Barbieri and Reuveny (2005), Fearon and Laitin (2003), and Gleditsch (1998) who find growing populations strain resources such as food, land, jobs, or infrastructure (population pressure hypothesis) leading to civil unrest and dissatisfaction with government. Tir and Diehl (1998) also finds that countries with lower technology levels are more prone to population pressures and conflict compared to other countries indicating that access to advanced technology may lessen the harmful effects of high population growth.

Education has no significant effect on state-based conflicts but appears to increase outcomes for both non state-based and civilian based conflicts. (Krueger and Maleckova (2003)) finds that violent and radical attacks do not decrease with higher education and living standards. Political knowledge and lower tolerance for oppressive regimes are more likely in populations with high levels of education resulting in increased civilian protests, as seen in South Africa during the Zuma regime and in Zimbabwe during the Mugabe regime. This argument supports the " $u$ " shaped non-linear relationship under state-based conflicts. However, the opposite holds for low intensity conflicts under groups and civilians. Initial low levels of education increase conflicts, mostly on the back of unemployed youth who become easy recruits for rebel groups. More educated people are better equipped for labour market which keeps youth off the streets (Collier and Hoeffler (2004a) and Reynal-Querol $(2002)) \cdot{ }^{11}$

\section{Additional analysis}

Several additional controls were used to check robustness of the results. The additional controls include geographical variables that measure terrain ruggedness and distance to the nearest coast, historical variables that capture the effects of slave trade (Nunn and Wantchekon (2011), Nunn (2008)), colonial rule in Africa (Acemoglu et al. (2001)), distance to the transatlantic and Indian Ocean slave trades, and initial income per capita in 1950. ${ }^{12}$ According to Nunn and Puga (2012), rugged terrain offers protection to the population from raids through increased hiding position and vantage points for scouting as well as increasing the cost of transportation of weaponry. ${ }^{13}$

We also decompose globalisation into its three components: political, economic and social. Political globalisation measures the number of embassies in the country, membership in international

\footnotetext{
${ }^{11}$ Results with secondary education can be found in Appendix B.

${ }^{12}$ The variables are from Nunn and Puga (2012) and downloaded from http://diegopuga.org/data/rugged/.

${ }^{13}$ Increased transportation costs is also true for countries farther from the coast.
} 
organisations, participation in United Nations (UN) Security Council missions and number of international treaties. Social globalisation measures personal contact and cultural proximity through tourism, the percentage of foreign population in countries, telephone traffic, internet use, media use, trade in books, and presence of multinational corporations. Economic globalisation measures trade and financial openness through actual flows of goods, foreign direct investment, foreign portfolio investment, income payments to foreign nationals, and trade restrictions such as capital account restrictions, hidden import barriers and mean tariff rates. The decomposition helps us to identify the type of globalisation driving the results.

Similarly, we decompose the state fragility index into effectiveness (ability to provide for population and control violence) and legitimacy (acceptance of rule by the population). Tables 3 to 6 report the results.

The additional geographic and historical controls do not change the overall interpretation of the initial results with globalisation and resource rents persistently increasing the number of conflicts across the three types. The positive globalisation result is driven by political globalisation while economic and social globalisation decreases conflict occurrences. This supports (Blanton and Apodaca, 2007), (Choi, 2010), (Flaten and de Soysa, 2012), and (Hegre et al., 2010) that pacifying effects are promoted by increased economic and social globalisation encouraging or at least mitigating conflicts. In contrast, the arms trade in Africa illustrates ways in which leaders can use their political networks to fund civil wars and unrest.

A lack of legitimacy of state powers contributes significantly to increasing the number of conflicts and is the primary driver of the positive state fragility effects. A lack of faith or conviction held by the population in a government's rule increases the incidence of conflicts. Examples include the increased civilian protests under President Zuma in South Africa, under President Kabila in the DRC, and under President Mugabe in Zimbabwe. These protests culminated in the fall of these leaders.

To summarize the remaining results, distance to the nearest coast increases all conflict types regardless of intensity and distance to atlantic slave trade routes increases only state-based conflicts. According to Nunn and Wantchekon (2011), people from countries where slave trade was most prominent are less trusting today. Rugged terrain increases low intensity violence across all three conflict types. While areas that are difficult to access can serve as an obstacle to conflict, rugged terrain can also create opportunities for ambush, fighting over land, and reduce government's ability to protect citizens. Being a former British colony reduces civilian-based conflicts in relation to other colonial types. Countries with low initial wealth in 1950 have increased conflicts today.

\section{Conclusion}

This paper highlights heterogeneity in determinants of conflict across types and intensity groupings in Africa. We find evidence of heterogeneity in income per capita, education and population density 
across conflict types. This is not the case of all determinants. We find that globalisation, military expenditure, resource rents and state fragility have consistent positive effects on all conflict types regardless of the intensity groups. These results remain robust to the inclusion of various controls and the inclusion of a breakdown of globalisation and state fragility.

While it is presumptuous to make policy recommendations based on these results, the preliminary findings that determinants of conflicts are unique to type of conflict should not be dismissed. Further research is required to determine the 'why' but the initial findings suggest that implementing long lasting solutions to mitigate conflict should focus on the nature of the conflict specific to a country. This in turn will hopefully feed into improving overall economic development. 


\section{References}

Acemoglu, D., S. Johnson, And J. Robinson (2001): "The Colonial Origins of Comparative Development: An Empirical Investigation," American Economic Review, 91, 1369-1401.

Arezki, R. And T. Gylfason (2013): "Resource Rents, Democracy, Corruption and Conflict: Evidence from Sub-Saharan Africa," Journal of African Economies, 22, 552-569.

Barbieri, K. And R. Reuveny (2005): "Economic Globalization and Civil War," Journal of Politics, 67, 1228-1247.

Bezemer, D. And R. Jong-A-Pin (2013): "Democracy, globalization and ethnic violence," Journal of Comparative Economics, 41, 108-125.

Blanton, R. and C. Apodaca (2007): "Economic Globalization and Violent Civil Conflict: Is Openness a Pathway to Peace?" The Social Science Journal, 44, 599-619.

Blattman, C. And E. Miguel (2010): "Civil War," Journal of Economic Literature, 48, 3-57.

Cameron, A. And P. Trivedi (2005): Microeconometrics: Methods and Applications, Cambridge University Press.

Chol, S.-W. (2010): "Beyond Kantian Liberalism," Conflict Management and Peace Science, 27, $272-295$.

Collier, P. And A. Hoeffler (1998): "On Economic Causes of Civil War," Oxford Economic Papers, 50, 563-573.

_ (2002): "On the Incidence of Civil War in Africa," Journal of Conflict Resolution, 46, 13-28.

- (2004a): "Greed and grievance in civil war," Oxford Economic Papers, 56, 563-595.

- (2004b): "Military Expenditure in Post-Conflict Societies," Development and comp systems, EconWPA.

Collier, P., A. Hoeffler, And D. Rohner (2009): "Beyond greed and grievance: feasibility and civil war," Oxford Economic Papers, 61, 1-27.

Collier, P., A. Hoeffler, And M. Soderbom (2004): "On the Duration of Civil War," Journal of Peace Research, 41, 253-273.

Drener, A. (2006): "Does globalization affect growth? Evidence from a new index of globalization," Applied Economics, 38, 1091-1110.

Dreher, A., N. Gaston, And P. Martens (2008): Measuring Globalisation: Gauging its Consequences, New York, Springer. 
Fearon, J. D. And D. D. Laitin (2003): "Ethnicity, Insurgency, and Civil War," American Political Science Review, 97, 75-90.

Flaten, R. D. And I. De Soysa (2012): “Globalization and Political Violence, 1970-2008," International Interactions, 38, 622-646.

Gleditsch, K. S. (2007): "Transnational Dimensions of Civil War," Journal of Peace Research, 44, 293-309.

Gleditsch, N. P. (1998): "Armed Conflict and The Environment: A Critique of the Literature," Journal of Peace Research, 35, 381-400.

Hegre, H., J. R. Oneal, And B. Russett (2010): "Trade does promote peace: New simultaneous estimates of the reciprocal effects of trade and conflict," Journal of Peace Research, 47, 763-774.

Hegre, H. And N. Sambanis (2006): "Sensitivity Analysis of Empirical Results on Civil War Onset," Journal of Conflict Resolution, 50, 508-535.

Krueger, A. B. And J. Maleckova (2003): "Education, Poverty and Terrorism: Is There a Causal Connection?" Journal of Economic Perspectives, 17, 119-144.

Michalopoulos, S. And E. Papaionnnou (2016): "The Long-Run Effects of the Scramble for Africa," Tech. Rep. 7.

Miguel, E., S. Satyanath, and E. Sergenti (2004): "Economic Shocks and Civil Conflict: An Instrumental Variables Approach," Journal of Political Economy, 112, 725-753.

Nunn, N. (2008): "The Long-term Effects of Africa's Slave Trades," The Quarterly Journal of Economics, 123, 139-176.

Nunn, N. And D. Puga (2012): "Ruggedness: The Blessing of Bad Geography in Africa," The Review of Economics and Statistics, 94, 20-36.

Nunn, N. And L. Wantchekon (2011): "The Slave Trade and the Origins of Mistrust in Africa," American Economic Review, 101, 3221-52.

OlzaK, S. (2011): "Does Globalization Breed Ethnic Discontent?" Journal of Conflict Resolution, $55,3-32$.

Pinker, S. (2011): The Better Angels of our Nature, New York, NY: Viking.

Raleigh, C., A. Linke, H. Hegre, and J. Karlsen (2010): "Introducing AClED: An Armed Conflict Location and Event Dataset," Journal of Peace Research, 47, 651-660.

Reynal-Querol, M. (2002): "Political systems, stability and civil wars," Defence and Peace Economics, 13, 465-483. 
Rouen, K. R. D. And D. Sobek (2004): "The Dynamics of Civil War Duration and Outcome," Journal of Peace Research, 41, 303-320.

TiR, J. And P. F. Diehl (1998): "Demographic Pressure and Interstate Conflict: Linking Population Growth and Density to Militarized Disputes and Wars, 1930-89," Journal of Peace Research, $35,319-339$.

Wooldridge, J. M. (2010): Econometric Analysis of Cross Section and Panel Data, vol. 1 of MIT Press Books, The MIT Press. 


\section{Figures \& Tables}

Figure 1: Intensity and Events: All Conflicts 1997-2017

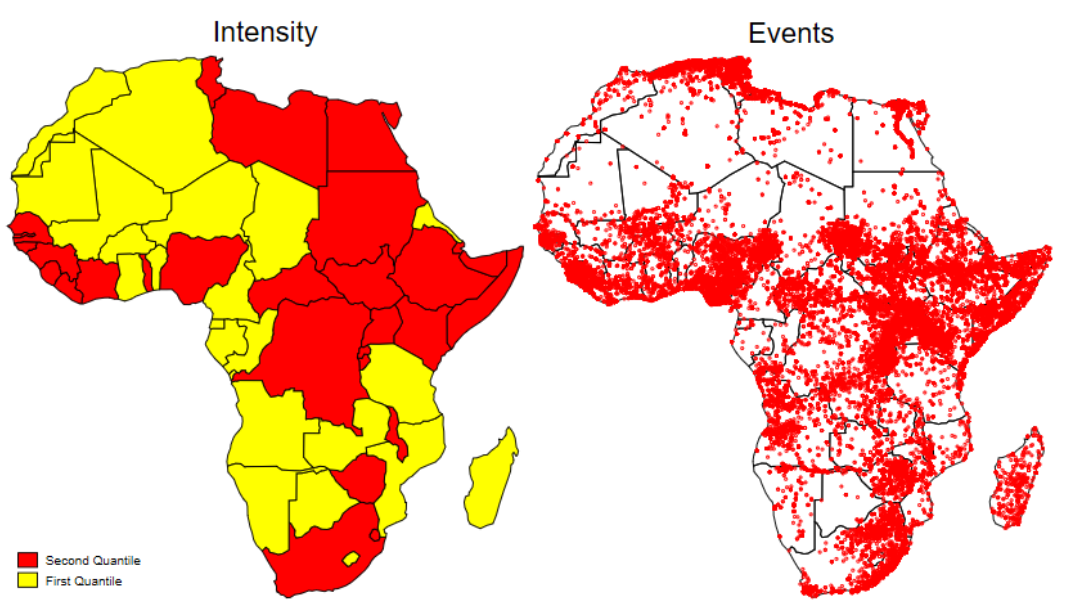

Note: Intensity is the total number of events over the entire sample divided by the square kilometer of the country. We have divided the intensity into 2 quantiles with the first quantile being $0-50 \%$.

Figure 2: Intensity and Events: Separted by Type 1997-2017

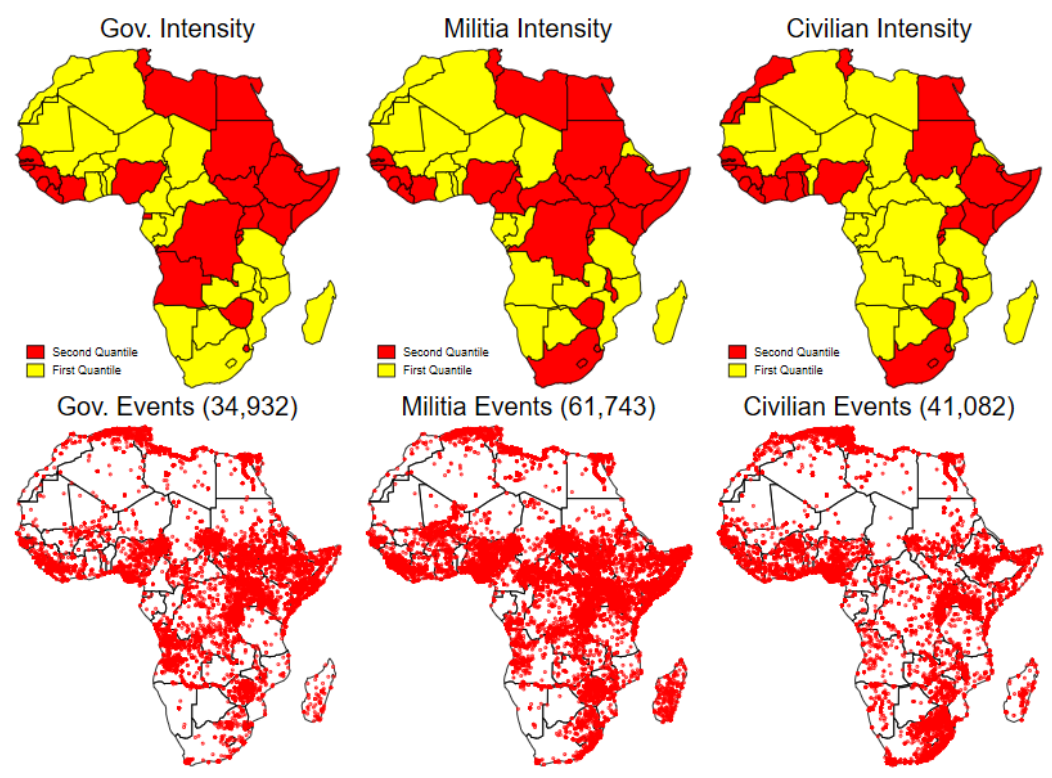

Note: Intensity is the total number of events over the entire sample divided by the square kilometer of the country. We have divided the intensity into 2 quantiles with the first quantile being $0-50 \%$. The count of events of each type are in parenthesis. 
Table 1: Negative Binomial

\begin{tabular}{|c|c|c|c|c|c|c|c|c|c|c|c|c|}
\hline \multirow[b]{2}{*}{ (1) } & \multicolumn{6}{|c|}{ All Conflicts } & \multicolumn{6}{|c|}{ Governments/Related Parties } \\
\hline & $\begin{array}{l}(2) \\
\text { All }\end{array}$ & $\begin{array}{l}(3) \\
\text { All Sq. }\end{array}$ & $\begin{array}{l}(4) \\
\text { LQ }\end{array}$ & $\begin{array}{c}(5) \\
\text { LQ Sq. }\end{array}$ & $\begin{array}{l}(6) \\
\mathrm{HQ} \\
\end{array}$ & $\begin{array}{c}(7) \\
\text { HQ Sq. }\end{array}$ & $\begin{array}{l}(8) \\
\text { All }\end{array}$ & $\begin{array}{c}(9) \\
\text { All Sq. }\end{array}$ & $\begin{array}{l}(10) \\
\text { LQ }\end{array}$ & $\begin{array}{c}(11) \\
\text { LQ Sq. }\end{array}$ & $\begin{array}{l}(12 \\
\mathrm{HQ} \\
\end{array}$ & $\begin{array}{l}(13) \\
\text { HQ Sq. }\end{array}$ \\
\hline $\ln ($ Military $\operatorname{Exp} \cdot(t-1))$ & $\begin{array}{c}0.548^{* * *} \\
(0.127)\end{array}$ & $\begin{array}{l}0.470^{* * *} \\
(0.169)\end{array}$ & $\begin{array}{l}0.961^{* * *} \\
(0.157)\end{array}$ & $\begin{array}{c}0.273 \\
(0.258)\end{array}$ & $\begin{array}{c}0.497^{* * *} \\
(0.161)\end{array}$ & $\begin{array}{c}0.068 \\
(0.224)\end{array}$ & $\begin{array}{c}0.576^{* * *} \\
(0.142)\end{array}$ & $\begin{array}{l}0.655^{* * *} \\
(0.213)\end{array}$ & $\begin{array}{l}0.401^{*} \\
(0.235)\end{array}$ & $\begin{array}{c}0.407 \\
(0.345)\end{array}$ & $\begin{array}{l}0.441^{* *} \\
(0.187)\end{array}$ & $\begin{array}{c}0.263 \\
(0.260)\end{array}$ \\
\hline $\ln ($ Military Exp.(t-1) $)$ Sq. & & $\begin{array}{c}-0.218^{* *} \\
(0.099)\end{array}$ & & $\begin{array}{c}-0.269^{* * *} \\
(0.103)\end{array}$ & & $\begin{array}{c}0.084 \\
(0.127)\end{array}$ & & $\begin{array}{l}-0.253^{*} \\
(0.134)\end{array}$ & & $\begin{array}{c}0.029 \\
(0.259)\end{array}$ & & $\begin{array}{l}-0.077 \\
(0.140)\end{array}$ \\
\hline $\ln \left(\right.$ Real $\left.\operatorname{PCGDP}_{(t-1)}\right)$ & $\begin{array}{l}0.236^{*} \\
(0.135)\end{array}$ & $\begin{array}{c}-3.600^{* * *} \\
(1.252)\end{array}$ & $\begin{array}{c}-0.419^{* *} \\
(0.175)\end{array}$ & $\begin{array}{c}3.878 \\
(2.509)\end{array}$ & $\begin{array}{l}0.356^{*} \\
(0.185)\end{array}$ & $\begin{array}{l}-1.981 \\
(1.581)\end{array}$ & $\begin{array}{l}0.291^{*} \\
(0.162)\end{array}$ & $\begin{array}{l}-2.771 \\
(1.823)\end{array}$ & $\begin{array}{l}0.478^{* *} \\
(0.187)\end{array}$ & $\begin{array}{l}-1.935 \\
(2.444)\end{array}$ & $\begin{array}{l}-0.033 \\
(0.211)\end{array}$ & $\begin{array}{l}-2.731 \\
(2.829)\end{array}$ \\
\hline $\ln ($ Real PCGDP $(t-1))$ Sq. & & $\begin{array}{c}0.257^{* * *} \\
(0.086)\end{array}$ & & $\begin{array}{l}-0.256 \\
(0.169)\end{array}$ & & $\begin{array}{c}0.140 \\
(0.109)\end{array}$ & & $\begin{array}{l}0.216^{*} \\
(0.128)\end{array}$ & & $\begin{array}{c}0.143 \\
(0.170)\end{array}$ & & $\begin{array}{c}0.202 \\
(0.203)\end{array}$ \\
\hline $\ln \left(\right.$ Globalisation $\left._{(t-1)}\right)$ & $\begin{array}{l}3.005^{* * *} \\
(0.614)\end{array}$ & $\begin{array}{c}-73.101^{* * *} \\
(10.211)\end{array}$ & $\begin{array}{l}4.231^{* * *} \\
(0.677)\end{array}$ & $\begin{array}{c}-75.155^{* * *} \\
(13.540)\end{array}$ & $\begin{array}{l}1.918^{* *} \\
(0.890)\end{array}$ & $\begin{array}{c}-64.366^{* * *} \\
(11.614)\end{array}$ & $\begin{array}{l}1.666^{* *} \\
(0.662)\end{array}$ & $\begin{array}{c}-66.719^{* * *} \\
(13.095)\end{array}$ & $\begin{array}{c}0.374 \\
(0.999)\end{array}$ & $\begin{array}{c}-96.035^{* * *} \\
(18.065)\end{array}$ & $\begin{array}{l}2.397^{* *} \\
(0.931)\end{array}$ & $\begin{array}{c}-39.523^{* * *} \\
(13.986)\end{array}$ \\
\hline $\ln \left(\right.$ Globalisation $\left._{(t-1)}\right)$ Sq. & & $\begin{array}{c}10.256^{* * *} \\
(1.349)\end{array}$ & & $\begin{array}{c}10.461^{* * *} \\
(1.774)\end{array}$ & & $\begin{array}{c}9.101^{* * *} \\
(1.514)\end{array}$ & & $\begin{array}{c}9.155^{* * *} \\
(1.746)\end{array}$ & & $\begin{array}{c}12.690^{* * *} \\
(2.410)\end{array}$ & & $\begin{array}{c}5.719^{* * *} \\
(1.892)\end{array}$ \\
\hline $\ln \left(\right.$ Resource Rents $\left._{(t-1)}\right)$ & $\begin{array}{c}0.750^{* * *} \\
(0.098)\end{array}$ & $\begin{array}{c}0.601^{* * *} \\
(0.159)\end{array}$ & $\begin{array}{c}0.504^{* * *} \\
(0.111)\end{array}$ & $\begin{array}{c}0.291 \\
(0.207)\end{array}$ & $\begin{array}{c}0.952^{* * *} \\
(0.130)\end{array}$ & $\begin{array}{c}1.190^{* * *} \\
(0.268)\end{array}$ & $\begin{array}{c}0.694^{* * *} \\
(0.117)\end{array}$ & $\begin{array}{l}0.284^{*} \\
(0.158)\end{array}$ & $\begin{array}{c}0.560^{* * *} \\
(0.154)\end{array}$ & $\begin{array}{l}-0.146 \\
(0.248)\end{array}$ & $\begin{array}{c}1.008^{* * *} \\
(0.130)\end{array}$ & $\begin{array}{c}1.366^{* * *} \\
(0.293)\end{array}$ \\
\hline $\ln \left(\right.$ Resource Rents $\left._{(t-1)}\right)$ Sq. & & $\begin{array}{c}-0.151^{* * *} \\
(0.039)\end{array}$ & & $\begin{array}{l}-0.001 \\
(0.063)\end{array}$ & & $\begin{array}{c}-0.276^{* * *} \\
(0.062)\end{array}$ & & $\begin{array}{l}-0.045 \\
(0.041)\end{array}$ & & $\begin{array}{c}0.095 \\
(0.069)\end{array}$ & & $\begin{array}{c}-0.262^{* * *} \\
(0.081)\end{array}$ \\
\hline $\ln \left(\right.$ Primary Educ. $\left._{(t-1)}\right)$ & $\begin{array}{c}0.080 \\
(0.313)\end{array}$ & $\begin{array}{l}-0.837 \\
(4.155)\end{array}$ & $\begin{array}{c}1.238^{* * *} \\
(0.404)\end{array}$ & $\begin{array}{l}-8.762 \\
(7.938)\end{array}$ & $\begin{array}{c}0.367 \\
(0.447)\end{array}$ & $\begin{array}{l}-4.402 \\
(5.399)\end{array}$ & $\begin{array}{l}-0.013 \\
(0.359)\end{array}$ & $\begin{array}{l}-0.567 \\
(4.543)\end{array}$ & $\begin{array}{c}0.462 \\
(0.472)\end{array}$ & $\begin{array}{l}-8.054 \\
(9.947)\end{array}$ & $\begin{array}{l}-0.110 \\
(0.444)\end{array}$ & $\begin{array}{c}-16.311^{* * *} \\
(6.270)\end{array}$ \\
\hline $\ln \left(\operatorname{Primary}\right.$ Educ. $\left._{(t-1)}\right)$ Sq. & & $\begin{array}{c}0.276 \\
(0.477)\end{array}$ & & $\begin{array}{c}1.067 \\
(0.895)\end{array}$ & & $\begin{array}{c}0.683 \\
(0.619)\end{array}$ & & $\begin{array}{c}0.228 \\
(0.520)\end{array}$ & & $\begin{array}{c}1.106 \\
(1.124)\end{array}$ & & $\begin{array}{c}1.965^{* * *} \\
(0.730)\end{array}$ \\
\hline $\operatorname{Ln}($ Pop. Density $(t-1))$ & $\begin{array}{c}0.301^{* * *} \\
(0.069)\end{array}$ & $\begin{array}{c}1.051^{* * *} \\
(0.318)\end{array}$ & $\begin{array}{l}-0.045 \\
(0.089)\end{array}$ & $\begin{array}{c}3.524^{* * *} \\
(0.661)\end{array}$ & $\begin{array}{c}0.015 \\
(0.111)\end{array}$ & $\begin{array}{c}0.724 \\
(0.611)\end{array}$ & $\begin{array}{c}0.264^{* * *} \\
(0.091)\end{array}$ & $\begin{array}{c}1.193^{* * *} \\
(0.389)\end{array}$ & $\begin{array}{l}-0.089 \\
(0.131)\end{array}$ & $\begin{array}{c}2.233^{* * *} \\
(0.481)\end{array}$ & $\begin{array}{l}-0.012 \\
(0.151)\end{array}$ & $\begin{array}{c}1.345 \\
(0.928)\end{array}$ \\
\hline $\operatorname{Ln}$ Pop. Density $(t-1))$ Sq. & & $\begin{array}{c}-0.151^{* * *} \\
(0.048)\end{array}$ & & $\begin{array}{c}-0.676^{* * *} \\
(0.125)\end{array}$ & & $\begin{array}{l}-0.124 \\
(0.085)\end{array}$ & & $\begin{array}{c}-0.158^{* * *} \\
(0.054)\end{array}$ & & $\begin{array}{c}-0.409^{* * *} \\
(0.084)\end{array}$ & & $\begin{array}{l}-0.198^{*} \\
(0.112)\end{array}$ \\
\hline $\ln ($ State Frag. $(t-1))$ & $\begin{array}{c}1.223^{* * *} \\
(0.328)\end{array}$ & $\begin{array}{c}6.051^{* * *} \\
(0.599)\end{array}$ & $\begin{array}{c}1.496^{* * *} \\
(0.388)\end{array}$ & $\begin{array}{l}-1.378 \\
(1.222)\end{array}$ & $\begin{array}{c}0.572 \\
(0.389)\end{array}$ & $\begin{array}{c}7.978^{* * *} \\
(0.876)\end{array}$ & $\begin{array}{c}1.597^{* * *} \\
(0.352)\end{array}$ & $\begin{array}{c}6.104^{* * *} \\
(0.864)\end{array}$ & $\begin{array}{c}0.718 \\
(0.622)\end{array}$ & $\begin{array}{l}-1.128 \\
(1.642)\end{array}$ & $\begin{array}{l}0.966^{* *} \\
(0.413)\end{array}$ & $\begin{array}{c}8.096^{* * *} \\
(1.100)\end{array}$ \\
\hline $\ln ($ State Frag. $(t-1))$ Sq. & & $\begin{array}{c}1.525^{* * *} \\
(0.295)\end{array}$ & & $\begin{array}{c}-1.383^{* * *} \\
(0.493)\end{array}$ & & $\begin{array}{c}3.123^{* * *} \\
(0.401)\end{array}$ & & $\begin{array}{c}1.369^{* * *} \\
(0.346)\end{array}$ & & $\begin{array}{c}-1.565^{* *} \\
(0.659)\end{array}$ & & $\begin{array}{c}2.941^{* * *} \\
(0.496)\end{array}$ \\
\hline lnalpha & $\begin{array}{c}0.707^{* * *} \\
(0.042)\end{array}$ & $\begin{array}{l}0.505^{* * *} \\
(0.049)\end{array}$ & $\begin{array}{c}0.482^{* * *} \\
(0.079)\end{array}$ & $\begin{array}{c}0.351^{* * *} \\
(0.097) \\
\end{array}$ & $\begin{array}{c}0.617^{* * *} \\
(0.054)\end{array}$ & $\begin{array}{c}0.360^{* * *} \\
(0.077)\end{array}$ & $\begin{array}{c}1.089^{* * *} \\
(0.056)\end{array}$ & $\begin{array}{l}0.982^{* * *} \\
(0.061)\end{array}$ & $\begin{array}{c}0.966^{* * *} \\
(0.091)\end{array}$ & $\begin{array}{c}0.726^{* * *} \\
(0.096)\end{array}$ & $\begin{array}{c}0.922^{* * *} \\
(0.070)\end{array}$ & $\begin{array}{c}0.768^{* * *} \\
(0.081)\end{array}$ \\
\hline LogLik & -3273.299 & -3186.496 & -1328.035 & -1301.947 & -1877.847 & -1819.587 & -2176.914 & -2142.084 & -875.685 & -840.759 & -1255.072 & -1228.628 \\
\hline Pseudo-R2 & 0.042 & 0.067 & 0.076 & 0.094 & 0.020 & 0.051 & 0.048 & 0.064 & 0.066 & 0.103 & 0.031 & 0.051 \\
\hline Obs & 664 & 664 & 334 & 334 & 330 & 330 & 664 & 664 & 355 & 355 & 309 & 309 \\
\hline
\end{tabular}

Robust Standard errors in parentheses. * $p<.10,{ }^{* *} p<.05,{ }^{* * *} p<.01$. Note that the headers "All", "LQ", and "HQ" indicate that all conflicts of that type were used, only the lower quantile was used, and only

the upper quatile was used in the regressions. 
Table 2: Negative Binomial

\begin{tabular}{|c|c|c|c|c|c|c|c|c|c|c|c|c|}
\hline \multirow[b]{2}{*}{ (1) } & \multicolumn{6}{|c|}{ Groups/Militias } & \multicolumn{6}{|c|}{ Civilians/Protests } \\
\hline & $\begin{array}{l}(2) \\
\text { All }\end{array}$ & $\begin{array}{c}(3) \\
\text { All Sq. }\end{array}$ & $\begin{array}{l}\text { (4) } \\
\mathrm{LQ} \\
\end{array}$ & $\begin{array}{c}(5) \\
\text { LQ Sq. }\end{array}$ & $\begin{array}{l}(6) \\
\mathrm{HQ}\end{array}$ & $\begin{array}{c}(7) \\
\text { HQ Sq. }\end{array}$ & $\begin{array}{l}(8) \\
\text { All } \\
\end{array}$ & $\begin{array}{c}(9) \\
\text { All Sq. }\end{array}$ & $\begin{array}{l}\text { (10) } \\
\text { LQ }\end{array}$ & $\begin{array}{c}(11) \\
\text { LQ Sq. }\end{array}$ & $\begin{array}{l}(12 \\
\mathrm{HQ} \\
\end{array}$ & $\begin{array}{l}(13) \\
\text { HQ Sq. }\end{array}$ \\
\hline $\ln ($ Military Exp.t-1) & $\begin{array}{c}0.467^{* * *} \\
(0.141)\end{array}$ & $\begin{array}{c}0.263 \\
(0.177)\end{array}$ & $\begin{array}{c}0.102 \\
(0.149)\end{array}$ & $\begin{array}{c}-0.751^{* * * *} \\
(0.228)\end{array}$ & $\begin{array}{c}0.766^{* * *} \\
(0.181)\end{array}$ & $\begin{array}{c}0.187 \\
(0.215)\end{array}$ & $\begin{array}{l}0.250^{*} \\
(0.147)\end{array}$ & $\begin{array}{c}0.146 \\
(0.251)\end{array}$ & $\begin{array}{c}1.178^{* * *} \\
(0.262)\end{array}$ & $\begin{array}{l}1.059^{* *} \\
(0.515)\end{array}$ & $\begin{array}{l}-0.015 \\
(0.266)\end{array}$ & $\begin{array}{l}-0.109 \\
(0.300)\end{array}$ \\
\hline $\ln ($ Military Exp.t-1) Sq. & & $\begin{array}{l}-0.189^{*} \\
(0.111)\end{array}$ & & $\begin{array}{c}0.026 \\
(0.101)\end{array}$ & & $\begin{array}{c}0.095 \\
(0.154)\end{array}$ & & $\begin{array}{l}-0.052 \\
(0.112)\end{array}$ & & $\begin{array}{l}-0.245 \\
(0.186)\end{array}$ & & $\begin{array}{l}-0.160 \\
(0.133)\end{array}$ \\
\hline $\ln \left(\right.$ Real $\left.\operatorname{PCGDP}_{(t-1)}\right)$ & $\begin{array}{c}0.166 \\
(0.145)\end{array}$ & $\begin{array}{l}-1.837 \\
(1.299)\end{array}$ & $\begin{array}{l}-0.168 \\
(0.170)\end{array}$ & $\begin{array}{c}7.211^{* * *} \\
(1.622)\end{array}$ & $\begin{array}{c}0.016 \\
(0.217)\end{array}$ & $\begin{array}{l}-1.778 \\
(1.781)\end{array}$ & $\begin{array}{l}-0.021 \\
(0.129)\end{array}$ & $\begin{array}{c}-5.424^{* * *} \\
(1.378)\end{array}$ & $\begin{array}{c}-1.107^{* * *} \\
(0.204)\end{array}$ & $\begin{array}{c}-9.572^{* * *} \\
(2.519)\end{array}$ & $\begin{array}{l}0.403^{* * *} \\
(0.154)\end{array}$ & $\begin{array}{c}-4.409^{* * *} \\
(1.611)\end{array}$ \\
\hline $\ln \left(\right.$ Real $\left.\operatorname{PCGDP}_{(t-1)}\right)$ Sq. & & $\begin{array}{c}0.124 \\
(0.090)\end{array}$ & & $\begin{array}{c}-0.476^{* * *} \\
(0.108)\end{array}$ & & $\begin{array}{c}0.114 \\
(0.123)\end{array}$ & & $\begin{array}{c}0.370^{* * *} \\
(0.093)\end{array}$ & & $\begin{array}{c}0.604^{* * *} \\
(0.172)\end{array}$ & & $\begin{array}{c}0.315^{* * *} \\
(0.109)\end{array}$ \\
\hline $\ln \left(\right.$ Globalisation $\left._{(t-1)}\right)$ & $\begin{array}{l}2.757^{* * *} \\
(0.696)\end{array}$ & $\begin{array}{c}-74.810^{* * *} \\
(11.456)\end{array}$ & $\begin{array}{l}3.104^{* * *} \\
(0.711)\end{array}$ & $\begin{array}{c}-43.744^{* * *} \\
(13.587)\end{array}$ & $\begin{array}{c}3.914^{* * *} \\
(1.026)\end{array}$ & $\begin{array}{c}-75.436^{* * *} \\
(13.551)\end{array}$ & $\begin{array}{c}5.523^{* * *} \\
(0.659)\end{array}$ & $\begin{array}{c}-41.394^{* * *} \\
(11.860)\end{array}$ & $\begin{array}{l}4.537^{* * *} \\
(0.938)\end{array}$ & $\begin{array}{c}-98.899^{* * *} \\
(21.941)\end{array}$ & $\begin{array}{c}3.946^{* * *} \\
(0.861)\end{array}$ & $\begin{array}{c}-24.247^{* *} \\
(12.290)\end{array}$ \\
\hline $\ln \left(\right.$ Globalisation $\left._{(t-1)}\right)$ Sq. & & $\begin{array}{c}10.474^{* * *} \\
(1.510)\end{array}$ & & $\begin{array}{c}6.114^{* * *} \\
(1.818)\end{array}$ & & $\begin{array}{c}10.826^{* * *} \\
(1.782)\end{array}$ & & $\begin{array}{c}6.298^{* * *} \\
(1.547)\end{array}$ & & $\begin{array}{c}13.912^{* * *} \\
(2.934)\end{array}$ & & $\begin{array}{l}4.018^{* *} \\
(1.607)\end{array}$ \\
\hline $\ln \left(\operatorname{Resource~Rents}_{(t-1)}\right)$ & $\begin{array}{c}0.459^{* * *} \\
(0.113)\end{array}$ & $\begin{array}{l}0.421^{* *} \\
(0.176)\end{array}$ & $\begin{array}{c}0.353^{* * *} \\
(0.108)\end{array}$ & $\begin{array}{c}0.396 \\
(0.248)\end{array}$ & $\begin{array}{l}0.628^{* * *} \\
(0.148)\end{array}$ & $\begin{array}{l}-0.288 \\
(0.661)\end{array}$ & $\begin{array}{l}0.773^{* * *} \\
(0.096)\end{array}$ & $\begin{array}{c}1.012^{* * *} \\
(0.165)\end{array}$ & $\begin{array}{l}0.688^{* * *} \\
(0.135)\end{array}$ & $\begin{array}{c}0.896^{* * *} \\
(0.249)\end{array}$ & $\begin{array}{l}0.793^{* * *} \\
(0.103)\end{array}$ & $\begin{array}{c}0.760^{* * *} \\
(0.231)\end{array}$ \\
\hline $\ln \left(\operatorname{Resource} \operatorname{Rents}_{(t-1)}\right)$ Sq. & & $\begin{array}{c}-0.163^{* * *} \\
(0.043)\end{array}$ & & $\begin{array}{l}-0.087 \\
(0.069)\end{array}$ & & $\begin{array}{l}-0.041 \\
(0.137)\end{array}$ & & $\begin{array}{c}-0.219^{* * *} \\
(0.043)\end{array}$ & & $\begin{array}{c}-0.155^{* *} \\
(0.065)\end{array}$ & & $\begin{array}{c}-0.153^{* * *} \\
(0.058)\end{array}$ \\
\hline $\ln ($ Primary Educ. $(t-1))$ & $\begin{array}{c}0.447 \\
(0.360)\end{array}$ & $\begin{array}{c}8.129 \\
(5.366)\end{array}$ & $\begin{array}{l}1.754^{* * *} \\
(0.371)\end{array}$ & $\begin{array}{c}10.645^{* *} \\
(5.430)\end{array}$ & $\begin{array}{l}-0.678 \\
(0.573)\end{array}$ & $\begin{array}{l}-0.802 \\
(9.762)\end{array}$ & $\begin{array}{c}0.443 \\
(0.363)\end{array}$ & $\begin{array}{l}-4.683 \\
(6.565)\end{array}$ & $\begin{array}{l}2.763^{* * *} \\
(0.707)\end{array}$ & $\begin{array}{c}37.983^{* * *} \\
(14.171)\end{array}$ & $\begin{array}{l}0.731^{*} \\
(0.427)\end{array}$ & $\begin{array}{l}-4.885 \\
(6.942)\end{array}$ \\
\hline $\ln \left(\operatorname{Primary}\right.$ Educ. $\left._{(t-1)}\right)$ Sq. & & $\begin{array}{l}-0.674 \\
(0.616)\end{array}$ & & $\begin{array}{l}-1.025^{*} \\
(0.623)\end{array}$ & & $\begin{array}{c}0.286 \\
(1.106)\end{array}$ & & $\begin{array}{c}0.684 \\
(0.738)\end{array}$ & & $\begin{array}{l}-3.884^{* *} \\
(1.558)\end{array}$ & & $\begin{array}{c}0.673 \\
(0.806)\end{array}$ \\
\hline $\operatorname{Ln}($ Pop. Density $(t-1))$ & $\begin{array}{c}0.265^{* * *} \\
(0.075)\end{array}$ & $\begin{array}{c}0.548 \\
(0.382)\end{array}$ & $\begin{array}{c}-0.286^{* * *} \\
(0.100)\end{array}$ & $\begin{array}{c}3.438^{* * *} \\
(0.613)\end{array}$ & $\begin{array}{c}0.099 \\
(0.115)\end{array}$ & $\begin{array}{l}-0.484 \\
(0.663)\end{array}$ & $\begin{array}{c}0.285^{* * *} \\
(0.087)\end{array}$ & $\begin{array}{c}1.297^{* * *} \\
(0.315)\end{array}$ & $\begin{array}{c}0.024 \\
(0.119)\end{array}$ & $\begin{array}{l}1.562^{* *} \\
(0.693)\end{array}$ & $\begin{array}{c}0.112 \\
(0.229)\end{array}$ & $\begin{array}{l}-1.528 \\
(2.142)\end{array}$ \\
\hline Ln(Pop. Density $(t-1))$ Sq. & & $\begin{array}{l}-0.089 \\
(0.056)\end{array}$ & & $\begin{array}{c}-0.712^{* * *} \\
(0.105)\end{array}$ & & $\begin{array}{c}0.035 \\
(0.091)\end{array}$ & & $\begin{array}{c}-0.172^{* * *} \\
(0.053)\end{array}$ & & $\begin{array}{l}-0.240^{*} \\
(0.144)\end{array}$ & & $\begin{array}{c}0.143 \\
(0.252)\end{array}$ \\
\hline $\ln ($ State Frag. $(t-1))$ & $\begin{array}{c}2.593^{* * *} \\
(0.312)\end{array}$ & $\begin{array}{c}7.927^{* * *} \\
(0.696)\end{array}$ & $\begin{array}{l}2.486^{* * *} \\
(0.326)\end{array}$ & $\begin{array}{l}-0.987 \\
(1.144)\end{array}$ & $\begin{array}{l}2.281^{* * *} \\
(0.429)\end{array}$ & $\begin{array}{c}11.355^{* * *} \\
(1.004)\end{array}$ & $\begin{array}{c}0.275 \\
(0.308)\end{array}$ & $\begin{array}{c}2.571^{* * *} \\
(0.824)\end{array}$ & $\begin{array}{c}0.018 \\
(0.454)\end{array}$ & $\begin{array}{l}-0.129 \\
(1.069)\end{array}$ & $\begin{array}{l}-0.043 \\
(0.363)\end{array}$ & $\begin{array}{l}4.184^{* * *} \\
(1.487)\end{array}$ \\
\hline $\ln ($ State Frag. $(t-1))$ Sq. & & $\begin{array}{c}1.913^{* * *} \\
(0.292)\end{array}$ & & $\begin{array}{c}-1.397^{* * *} \\
(0.456)\end{array}$ & & $\begin{array}{c}3.905^{* * *} \\
(0.459)\end{array}$ & & $\begin{array}{c}0.549 \\
(0.425)\end{array}$ & & $\begin{array}{l}-0.781^{*} \\
(0.417)\end{array}$ & & $\begin{array}{l}1.684^{* *} \\
(0.668)\end{array}$ \\
\hline lnalpha & $\begin{array}{l}1.019^{* * *} \\
(0.049)\end{array}$ & $\begin{array}{c}0.846^{* * *} \\
(0.054)\end{array}$ & $\begin{array}{l}0.783^{* * *} \\
(0.088)\end{array}$ & $\begin{array}{l}0.555^{* * *} \\
(0.090)\end{array}$ & $\begin{array}{l}0.932^{* * *} \\
(0.059)\end{array}$ & $\begin{array}{c}0.703^{* * *} \\
(0.071)\end{array}$ & $\begin{array}{c}0.878^{* * *} \\
(0.068)\end{array}$ & $\begin{array}{c}0.732^{* * *} \\
(0.073)\end{array}$ & $\begin{array}{c}0.691^{* * *} \\
(0.098)\end{array}$ & $\begin{array}{c}0.501^{* * *} \\
(0.100)\end{array}$ & $\begin{array}{l}0.798^{* * *} \\
(0.087)\end{array}$ & $\begin{array}{c}0.712^{* * *} \\
(0.095)\end{array}$ \\
\hline LogLik & -2507.895 & -2445.201 & -893.216 & -863.442 & -1561.238 & -1513.328 & -2432.951 & -2378.178 & -839.546 & -814.187 & -1546.079 & -1527.975 \\
\hline Pseudo-R2 & 0.048 & 0.071 & 0.084 & 0.114 & 0.024 & 0.054 & 0.066 & 0.087 & 0.095 & 0.123 & 0.060 & 0.071 \\
\hline Obs & 664 & 664 & 320 & 320 & 344 & 344 & 664 & 664 & 286 & 286 & 378 & 378 \\
\hline
\end{tabular}

the upper quatile was used in the regressions. 
Table 3: Negative Binomial: All Conflict

\begin{tabular}{|c|c|c|c|c|c|c|c|c|c|}
\hline \multirow[b]{2}{*}{ (1) } & \multicolumn{3}{|c|}{ Globalisation } & \multicolumn{3}{|c|}{ Fragility } & \multicolumn{3}{|c|}{ Geographic } \\
\hline & $\begin{array}{l}(2) \\
\text { All }\end{array}$ & $\begin{array}{l}(3) \\
\text { LQ }\end{array}$ & $\begin{array}{l}(4) \\
\mathrm{HQ}\end{array}$ & $\begin{array}{l}(5) \\
\text { All }\end{array}$ & $\begin{array}{l}(6) \\
\mathrm{LQ}\end{array}$ & $\begin{array}{l}(7) \\
\mathrm{HQ} \\
\end{array}$ & $\begin{array}{l}(8) \\
\text { All }\end{array}$ & $\begin{array}{l}(9) \\
\text { LQ }\end{array}$ & $\begin{array}{l}(10) \\
\mathrm{HQ}\end{array}$ \\
\hline $\ln ($ Military Exp.t-1) & $\begin{array}{c}0.658^{* * *} \\
(0.096)\end{array}$ & $\begin{array}{l}0.691 * * * \\
(0.184)\end{array}$ & $\begin{array}{l}0.524^{* * *} \\
(0.103)\end{array}$ & $\begin{array}{l}0.454^{* * *} \\
(0.128)\end{array}$ & $\begin{array}{l}0.962^{* * *} \\
(0.161)\end{array}$ & $\begin{array}{l}0.500^{* * *} \\
(0.145)\end{array}$ & $\begin{array}{l}0.179 \\
(0.138)\end{array}$ & $\begin{array}{c}0.068 \\
(0.262)\end{array}$ & $\begin{array}{l}0.656^{* * *} \\
(0.178)\end{array}$ \\
\hline $\ln \left(\right.$ Real $\left.\operatorname{PCGDP}_{(t-1)}\right)$ & $\begin{array}{c}0.444^{* * *} \\
(0.120)\end{array}$ & $\begin{array}{l}-0.087 \\
(0.165)\end{array}$ & $\begin{array}{c}0.544^{* * *} \\
(0.135)\end{array}$ & $\begin{array}{l}-0.013 \\
(0.152)\end{array}$ & $\begin{array}{l}-0.379 \\
(0.242)\end{array}$ & $\begin{array}{c}0.084 \\
(0.195)\end{array}$ & $\begin{array}{c}-0.460^{* * *} \\
(0.149)\end{array}$ & $\begin{array}{c}-0.481^{* *} \\
(0.203)\end{array}$ & $\begin{array}{c}-0.988^{* * *} \\
(0.254)\end{array}$ \\
\hline $\ln \left(\right.$ Globalisation $\left._{(t-1)}\right)$ & & & & $\begin{array}{l}2.417^{* * *} \\
(0.636)\end{array}$ & $\begin{array}{l}4.050^{* * *} \\
(0.693)\end{array}$ & $\begin{array}{l}1.948^{* *} \\
(0.827)\end{array}$ & $\begin{array}{l}3.056^{* * *} \\
(0.588)\end{array}$ & $\begin{array}{l}4.718^{* * *} \\
(0.787)\end{array}$ & $\begin{array}{c}5.353^{* * *} \\
(0.642)\end{array}$ \\
\hline $\ln ($ Econ. Globalisation $(t-1))$ & $\begin{array}{c}-0.861^{* * *} \\
(0.324)\end{array}$ & $\begin{array}{c}1.120 \\
(0.710)\end{array}$ & $\begin{array}{c}-1.458^{* * *} \\
(0.352)\end{array}$ & & & & & & \\
\hline $\ln ($ Social Globalisation $(t-1))$ & $\begin{array}{c}-1.156^{* * *} \\
(0.368)\end{array}$ & $\begin{array}{c}-1.707^{* * *} \\
(0.657)\end{array}$ & $\begin{array}{c}-1.195^{* * *} \\
(0.408)\end{array}$ & & & & & & \\
\hline $\ln ($ Pol. Globalisation $(t-1))$ & $\begin{array}{c}3.670^{* * *} \\
(0.236)\end{array}$ & $\begin{array}{c}4.482^{* * *} \\
(0.500)\end{array}$ & $\begin{array}{c}3.350^{* * *} \\
(0.286)\end{array}$ & & & & & & \\
\hline $\ln \left(\operatorname{Resource} \operatorname{Rents}_{(t-1)}\right)$ & $\begin{array}{l}0.425^{* * *} \\
(0.079)\end{array}$ & $\begin{array}{c}0.316^{* * *} \\
(0.116)\end{array}$ & $\begin{array}{l}0.474^{* * *} \\
(0.095)\end{array}$ & $\begin{array}{l}0.595^{* * *} \\
(0.107)\end{array}$ & $\begin{array}{c}0.536^{* * *} \\
(0.124)\end{array}$ & $\begin{array}{c}0.833^{* * *} \\
(0.135)\end{array}$ & $\begin{array}{c}0.708^{* * *} \\
(0.098)\end{array}$ & $\begin{array}{l}0.535^{* * *} \\
(0.118)\end{array}$ & $\begin{array}{l}-0.008 \\
(0.157)\end{array}$ \\
\hline $\ln ($ Primary Educ. $(t-1))$ & $\begin{array}{l}0.658^{* *} \\
(0.290)\end{array}$ & $\begin{array}{c}1.873^{* * *} \\
(0.399)\end{array}$ & $\begin{array}{l}0.589^{*} \\
(0.353)\end{array}$ & $\begin{array}{c}0.425 \\
(0.321)\end{array}$ & $\begin{array}{c}1.332^{* * *} \\
(0.424)\end{array}$ & $\begin{array}{c}0.589 \\
(0.441)\end{array}$ & $\begin{array}{c}-1.060^{* * *} \\
(0.399)\end{array}$ & $\begin{array}{l}-0.327 \\
(0.489)\end{array}$ & $\begin{array}{c}-3.370^{* * *} \\
(0.455)\end{array}$ \\
\hline $\operatorname{Ln}($ Pop. Density $(t-1))$ & $\begin{array}{c}0.197^{* * *} \\
(0.057)\end{array}$ & $\begin{array}{c}-0.269^{* * *} \\
(0.088)\end{array}$ & $\begin{array}{c}0.077 \\
(0.087)\end{array}$ & $\begin{array}{c}0.324^{* * *} \\
(0.071)\end{array}$ & $\begin{array}{l}-0.049 \\
(0.103)\end{array}$ & $\begin{array}{c}0.027 \\
(0.111)\end{array}$ & $\begin{array}{c}0.289^{* * *} \\
(0.080)\end{array}$ & $\begin{array}{l}0.148 \\
(0.102)\end{array}$ & $\begin{array}{l}0.074 \\
(0.109)\end{array}$ \\
\hline $\ln ($ State Frag. $(t-1))$ & $\begin{array}{l}0.526^{* *} \\
(0.263)\end{array}$ & $\begin{array}{c}0.411 \\
(0.402)\end{array}$ & $\begin{array}{c}0.070 \\
(0.308)\end{array}$ & & & & $\begin{array}{c}0.177 \\
(0.350)\end{array}$ & $\begin{array}{c}0.365 \\
(0.364)\end{array}$ & $\begin{array}{c}-2.233^{* * *} \\
(0.394)\end{array}$ \\
\hline $\ln \left(\right.$ Effectiveness $\left._{(t-1)}\right)$ & & & & $\begin{array}{l}-0.439 \\
(0.377)\end{array}$ & $\begin{array}{c}0.881 \\
(0.582)\end{array}$ & $\begin{array}{l}-0.924^{*} \\
(0.503)\end{array}$ & & & \\
\hline $\ln \left(\operatorname{Legitimacy}_{(t-1)}\right)$ & & & & $\begin{array}{c}1.257^{* * *} \\
(0.254)\end{array}$ & $\begin{array}{l}0.540^{*} \\
(0.288)\end{array}$ & $\begin{array}{c}1.327^{* * *} \\
(0.412)\end{array}$ & & & \\
\hline $\ln$ (Ruggedness) & & & & & & & $\begin{array}{c}0.181^{* * *} \\
(0.055)\end{array}$ & $\begin{array}{l}0.389^{* * *} \\
(0.057)\end{array}$ & $\begin{array}{r}0.290^{* * *} \\
(0.101)\end{array}$ \\
\hline ln(Real PCGDP 1950) & & & & & & & $\begin{array}{c}1.165^{* * *} \\
(0.160)\end{array}$ & $\begin{array}{c}1.348^{* * *} \\
(0.205)\end{array}$ & $\begin{array}{c}0.307 \\
(0.240)\end{array}$ \\
\hline $\ln$ (Dist. Coast) & & & & & & & $\begin{array}{c}0.620^{* * *} \\
(0.148)\end{array}$ & $\begin{array}{c}1.395^{* * *} \\
(0.235)\end{array}$ & $\begin{array}{l}2.107^{* * *} \\
(0.154)\end{array}$ \\
\hline $\ln$ (Dist. Atl. Slave) & & & & & & & $\begin{array}{l}1.024^{* * *} \\
(0.283)\end{array}$ & $\begin{array}{c}0.092 \\
(0.599)\end{array}$ & $\begin{array}{l}-0.242 \\
(0.317)\end{array}$ \\
\hline ln(Dist. Indian Slave) & & & & & & & $\begin{array}{l}0.297^{*} \\
(0.154)\end{array}$ & $\begin{array}{c}-0.497^{* *} \\
(0.204)\end{array}$ & $\begin{array}{c}1.374^{* * *} \\
(0.327)\end{array}$ \\
\hline British Colony & & & & & & & $\begin{array}{c}0.231 \\
(0.324)\end{array}$ & $\begin{array}{c}-2.506^{* *} \\
(1.139)\end{array}$ & $\begin{array}{l}0.870^{* *} \\
(0.408)\end{array}$ \\
\hline France Colony & & & & & & & $\begin{array}{l}-0.073 \\
(0.296)\end{array}$ & $\begin{array}{l}-1.591 \\
(1.115)\end{array}$ & $\begin{array}{c}-1.331^{* * *} \\
(0.419)\end{array}$ \\
\hline Portugal Colony & & & & & & & $\begin{array}{c}0.460 \\
(0.607) \\
\end{array}$ & $\begin{array}{r}-1.518 \\
(1.359) \\
\end{array}$ & $\begin{array}{c}3.948^{* * *} \\
(0.962) \\
\end{array}$ \\
\hline Inalpha & $\begin{array}{c}0.476^{* * *} \\
(0.044)\end{array}$ & $\begin{array}{c}0.270^{* * *} \\
(0.073)\end{array}$ & $\begin{array}{l}0.340^{* * *} \\
(0.065)\end{array}$ & $\begin{array}{l}0.685^{* * *} \\
(0.041)\end{array}$ & $\begin{array}{c}0.486^{* * *} \\
(0.078)\end{array}$ & $\begin{array}{c}0.595^{* * *} \\
(0.057)\end{array}$ & $\begin{array}{c}0.498^{* * *} \\
(0.047)\end{array}$ & $\begin{array}{l}0.174^{*} \\
(0.095) \\
\end{array}$ & $\begin{array}{l}0.185^{* *} \\
(0.075)\end{array}$ \\
\hline LogLik & -3153.139 & -1264.872 & -1815.422 & -3262.416 & -1328.697 & -1872.821 & -2977.547 & -1246.160 & -1609.581 \\
\hline $\begin{array}{l}\text { Pseudo-R2 } \\
\text { Obs }\end{array}$ & $\begin{array}{c}0.070 \\
658\end{array}$ & $\begin{array}{c}0.106 \\
328\end{array}$ & $\begin{array}{c}0.053 \\
330\end{array}$ & $\begin{array}{c}0.034 \\
645\end{array}$ & $\begin{array}{c}0.052 \\
315\end{array}$ & $\begin{array}{c}0.023 \\
330\end{array}$ & $\begin{array}{c}0.071 \\
623\end{array}$ & $\begin{array}{c}0.119 \\
328\end{array}$ & $\begin{array}{c}0.069 \\
295\end{array}$ \\
\hline
\end{tabular}


Table 4: Negative Binomial: Government/Related Parties

\begin{tabular}{|c|c|c|c|c|c|c|c|c|c|}
\hline \multirow[b]{2}{*}{ (1) } & \multicolumn{3}{|c|}{ Globalisation } & \multicolumn{3}{|c|}{ Fragility } & \multicolumn{3}{|c|}{ Geographic } \\
\hline & $\begin{array}{l}\text { (2) } \\
\text { All }\end{array}$ & $\begin{array}{l}(3) \\
\text { LQ }\end{array}$ & $\begin{array}{l}\text { (4) } \\
\text { HQ }\end{array}$ & $\begin{array}{l}\text { (5) } \\
\text { All }\end{array}$ & $\begin{array}{l}(6) \\
\text { LQ }\end{array}$ & $\begin{array}{l}\text { (7) } \\
\text { HQ }\end{array}$ & $\begin{array}{l}\text { (8) } \\
\text { All }\end{array}$ & $\begin{array}{l}(9) \\
\text { LQ }\end{array}$ & $\begin{array}{l}(10) \\
\text { HQ }\end{array}$ \\
\hline $\ln ($ Military Exp. $t-1)$ & $\begin{array}{c}0.710^{* * *} \\
(0.121)\end{array}$ & $\begin{array}{c}0.084 \\
(0.237)\end{array}$ & $\begin{array}{c}0.581^{* * *} \\
(0.142)\end{array}$ & $\begin{array}{c}0.541^{* * *} \\
(0.138)\end{array}$ & $\begin{array}{l}\begin{array}{c}0.532^{* *} \\
(0.217)\end{array} \\
\end{array}$ & $\begin{array}{l}0.414^{* *} \\
(0.167)\end{array}$ & $\begin{array}{c}0.075 \\
(0.179)\end{array}$ & $\begin{array}{c}-1.016^{* * *} \\
(0.285)\end{array}$ & $\begin{array}{c}-0.100 \\
(0.335)\end{array}$ \\
\hline $\ln \left(\right.$ Real $\left.\operatorname{PCGDP}_{(t-1)}\right)$ & $\begin{array}{c}0.629^{* * *} \\
(0.158)\end{array}$ & $\begin{array}{c}0.253 \\
(0.190)\end{array}$ & $\begin{array}{l}0.514^{* *} \\
(0.208)\end{array}$ & $\begin{array}{c}0.135 \\
(0.192)\end{array}$ & $\begin{array}{c}0.207 \\
(0.233)\end{array}$ & $\begin{array}{l}-0.239 \\
(0.212)\end{array}$ & $\begin{array}{c}-0.312 \\
(0.190)\end{array}$ & $\begin{array}{c}-1.468 * * * \\
(0.256)\end{array}$ & $\begin{array}{l}-0.241 \\
(0.199)\end{array}$ \\
\hline $\ln \left(\right.$ Globalisation $\left._{(t-1)}\right)$ & & & & $\begin{array}{l}1.292^{*} \\
(0.683)\end{array}$ & $\begin{array}{l}-0.242 \\
(0.992)\end{array}$ & $\begin{array}{l}1.907^{* *} \\
(0.948)\end{array}$ & $\begin{array}{l}1.754^{* *} \\
(0.723)\end{array}$ & $\begin{array}{l}4.931 * * * \\
(1.020)\end{array}$ & $\begin{array}{c}1.861 \\
(1.225)\end{array}$ \\
\hline $\ln \left(\right.$ Econ. Globalisation $\left.{ }_{(t-1)}\right)$ & $\begin{array}{c}-0.798^{* *} \\
(0.388)\end{array}$ & $\begin{array}{c}-2.692^{* * *} \\
(0.650)\end{array}$ & $\begin{array}{l}-0.804^{*} \\
(0.475)\end{array}$ & & & & & & \\
\hline $\ln ($ Social Globalisation $(t-1)$ & $\begin{array}{c}-2.113^{* * * *} \\
(0.488)\end{array}$ & $\begin{array}{l}-0.332 \\
(0.664)\end{array}$ & $\begin{array}{c}-2.323^{* * *} \\
(0.613)\end{array}$ & & & & & & \\
\hline $\ln ($ Pol. Globalisation $)$ & $\begin{array}{c}2.907^{* * *} \\
(0.342)\end{array}$ & $\begin{array}{c}3.582^{* * *} \\
(0.579)\end{array}$ & $\begin{array}{c}3.145^{* * *} \\
(0.435)\end{array}$ & & & & & & \\
\hline $\ln \left(\operatorname{Resource} \operatorname{Rents}_{(t-1)}\right)$ & $\begin{array}{c}0.309^{* * *} \\
(0.111)\end{array}$ & $\begin{array}{c}0.414^{* * *} \\
(0.150)\end{array}$ & $\begin{array}{c}0.370^{* * *} \\
(0.135)\end{array}$ & $\begin{array}{c}0.605^{* * *} \\
(0.132)\end{array}$ & $\begin{array}{c}0.545^{* * *} \\
(0.148)\end{array}$ & $\begin{array}{c}0.872^{* * *} \\
(0.136)\end{array}$ & $\begin{array}{c}0.560^{* * *} \\
(0.119)\end{array}$ & $\begin{array}{l}0.303^{* *} \\
(0.139)\end{array}$ & $\begin{array}{l}0.413^{*} \\
(0.211)\end{array}$ \\
\hline $\ln ($ Primary Educ.(t-1) $)$ & $\begin{array}{l}0.708^{*} \\
(0.404)\end{array}$ & $\begin{array}{l}1.208^{* *} \\
(0.471)\end{array}$ & $\begin{array}{c}0.647 \\
(0.469)\end{array}$ & $\begin{array}{c}0.215 \\
(0.370)\end{array}$ & $\begin{array}{c}0.571 \\
(0.451)\end{array}$ & $\begin{array}{c}0.284 \\
(0.457)\end{array}$ & $\begin{array}{c}-1.198^{* * * *} \\
(0.424)\end{array}$ & $\begin{array}{c}0.016 \\
(0.544)\end{array}$ & $\begin{array}{c}-2.171^{* * *} \\
(0.616)\end{array}$ \\
\hline Ln(Pop. Density $(t-1))$ & $\begin{array}{c}0.254^{* * *} \\
(0.083)\end{array}$ & $\begin{array}{c}-0.542^{* * *} \\
(0.132)\end{array}$ & $\begin{array}{c}0.190 \\
(0.142)\end{array}$ & $\begin{array}{c}0.275^{* * *} \\
(0.092)\end{array}$ & $\begin{array}{l}-0.084 \\
(0.142)\end{array}$ & $\begin{array}{l}-0.024 \\
(0.151)\end{array}$ & $\begin{array}{l}0.197^{* *} \\
(0.096)\end{array}$ & $\begin{array}{c}-0.412^{* * *} \\
(0.148)\end{array}$ & $\begin{array}{c}0.039 \\
(0.136)\end{array}$ \\
\hline $\ln ($ State Frag. $\cdot(t-1))$ & $\begin{array}{l}0.789^{* *} \\
(0.320)\end{array}$ & $\begin{array}{l}-0.151 \\
(0.615)\end{array}$ & $\begin{array}{c}0.291 \\
(0.371)\end{array}$ & & & & $\begin{array}{l}0.771 * * \\
(0.358)\end{array}$ & $\begin{array}{c}-0.990^{* *} \\
(0.478)\end{array}$ & $\begin{array}{c}-1.410^{* * *} \\
(0.514)\end{array}$ \\
\hline $\ln \left(\right.$ Effectiveness $\left._{(t-1)}\right)$ & & & & $\begin{array}{c}0.055 \\
(0.440)\end{array}$ & $\begin{array}{l}-0.530 \\
(0.716)\end{array}$ & $\begin{array}{c}-1.327^{* *} \\
(0.590)\end{array}$ & & & \\
\hline $\ln \left(\right.$ Legitimacy $\left._{(t-1)}\right)$ & & & & $\begin{array}{c}1.254^{* * *} \\
(0.280)\end{array}$ & $\begin{array}{c}0.460 \\
(0.330)\end{array}$ & $\begin{array}{c}1.895^{* * *} \\
(0.409)\end{array}$ & & & \\
\hline ln(Ruggedness) & & & & & & & $\begin{array}{c}0.219^{* * *} \\
(0.064)\end{array}$ & $\begin{array}{c}0.484^{* * *} \\
(0.071)\end{array}$ & $\begin{array}{c}-0.077 \\
(0.118)\end{array}$ \\
\hline $\ln$ (Real PCGDP 1950) & & & & & & & $\begin{array}{c}1.090^{* * *} \\
(0.223)\end{array}$ & $\begin{array}{c}2.348^{* * *} \\
(0.297)\end{array}$ & $\begin{array}{c}0.292 \\
(0.338)\end{array}$ \\
\hline $\ln$ (Dist. Coast) & & & & & & & $\begin{array}{c}0.508 * * * \\
(0.182)\end{array}$ & $\begin{array}{c}2.323^{* * *} \\
(0.294)\end{array}$ & $\begin{array}{c}1.571^{* * *} \\
(0.153)\end{array}$ \\
\hline $\ln$ (Dist. Atl. Slave) & & & & & & & $\begin{array}{c}1.515^{* * *} \\
(0.346)\end{array}$ & $\begin{array}{c}3.416 * * * \\
(0.736)\end{array}$ & $\begin{array}{l}0.950^{*} \\
(0.498)\end{array}$ \\
\hline $\ln$ (Dist. Indian Slave) & & & & & & & $\begin{array}{c}0.639^{* * *} \\
(0.215)\end{array}$ & $\begin{array}{l}0.627^{*} \\
(0.332)\end{array}$ & $\begin{array}{c}0.091 \\
(0.462)\end{array}$ \\
\hline British Colony & & & & & & & $\begin{array}{c}0.026 \\
(0.381)\end{array}$ & $\begin{array}{l}1.058^{* *} \\
(0.443)\end{array}$ & $\begin{array}{c}0.354 \\
(0.377)\end{array}$ \\
\hline France Colony & & & & & & & $\begin{array}{l}-0.343 \\
(0.331)\end{array}$ & $\begin{array}{c}2.998^{* * *} \\
(0.417)\end{array}$ & $\begin{array}{c}0.159 \\
(0.648)\end{array}$ \\
\hline Portugal Colony & & & & & & & $\begin{array}{c}1.101 \\
(0.749)\end{array}$ & $\begin{array}{c}0.000 \\
(.)\end{array}$ & $\begin{array}{c}2.743^{* * *} \\
(0.762)\end{array}$ \\
\hline lnalpha & $\begin{array}{c}0.916^{* * *} \\
(0.061)\end{array}$ & $\begin{array}{c}0.789^{* * *} \\
(0.094)\end{array}$ & $\begin{array}{c}0.688^{* * *} \\
(0.082)\end{array}$ & $\begin{array}{c}1.077^{* * *} \\
(0.056)\end{array}$ & $\begin{array}{c}0.963^{* * *} \\
(0.087)\end{array}$ & $\begin{array}{c}0.878^{* * *} \\
(0.073)\end{array}$ & $\begin{array}{c}0.924^{* * *} \\
(0.056)\end{array}$ & $\begin{array}{c}0.389^{* * *} \\
(0.109)\end{array}$ & $\begin{array}{c}0.663^{* * *} \\
(0.086)\end{array}$ \\
\hline LogLik & -2101.817 & -848.413 & -1196.870 & -2172.661 & -874.911 & -1247.311 & -1947.098 & -795.623 & -1043.262 \\
\hline Pseudo-R2 & 0.073 & 0.095 & 0.061 & 0.041 & 0.049 & 0.037 & 0.075 & 0.151 & 0.065 \\
\hline Obs & 658 & 355 & 303 & 645 & 336 & 309 & 623 & 355 & 268 \\
\hline
\end{tabular}


Table 5: Negative Binomial: Groups/Militias

\begin{tabular}{|c|c|c|c|c|c|c|c|c|c|}
\hline \multirow[b]{2}{*}{ (1) } & \multicolumn{3}{|c|}{ Globalisation } & \multicolumn{3}{|c|}{ Fragility } & \multicolumn{3}{|c|}{ Geographic } \\
\hline & $\begin{array}{l}(2) \\
\text { All }\end{array}$ & $\begin{array}{l}(3) \\
\text { LQ }\end{array}$ & $\begin{array}{l}(4) \\
\mathrm{HQ}\end{array}$ & $\begin{array}{l}(5) \\
\text { All }\end{array}$ & $\begin{array}{l}\text { (6) } \\
\text { LQ }\end{array}$ & $\begin{array}{l}\text { (7) } \\
\text { HQ }\end{array}$ & $\begin{array}{l}(8) \\
\text { All }\end{array}$ & $\begin{array}{l}(9) \\
\text { LQ }\end{array}$ & $\begin{array}{l}(10) \\
\mathrm{HQ}\end{array}$ \\
\hline $\ln ($ Military Exp. $t-1)$ & $\begin{array}{l}0.548^{* * *} \\
(0.110)\end{array}$ & $\begin{array}{l}-0.435^{* *} \\
(0.214)\end{array}$ & $\begin{array}{l}0.661^{* * *} \\
(0.125)\end{array}$ & $\begin{array}{l}0.347^{* *} \\
(0.139)\end{array}$ & $\begin{array}{c}0.104 \\
(0.151)\end{array}$ & $\begin{array}{l}0.760^{* * *} \\
(0.171)\end{array}$ & $\begin{array}{l}-0.010 \\
(0.167)\end{array}$ & $\begin{array}{l}-1.135^{* * *} \\
(0.370)\end{array}$ & $\begin{array}{l}0.602^{* *} \\
(0.253)\end{array}$ \\
\hline $\ln \left(\right.$ Real $\left.\operatorname{PCGDP}_{(t-1)}\right)$ & $\begin{array}{c}0.349^{* * *} \\
(0.128)\end{array}$ & $\begin{array}{c}0.147 \\
(0.207)\end{array}$ & $\begin{array}{c}0.445^{* * *} \\
(0.155)\end{array}$ & $\begin{array}{l}-0.093 \\
(0.157)\end{array}$ & $\begin{array}{l}-0.200 \\
(0.203)\end{array}$ & $\begin{array}{l}-0.231 \\
(0.221)\end{array}$ & $\begin{array}{c}-0.500^{* * * *} \\
(0.162)\end{array}$ & $\begin{array}{l}-0.073 \\
(0.234)\end{array}$ & $\begin{array}{c}-0.998^{* * *} \\
(0.291)\end{array}$ \\
\hline $\ln \left(\right.$ Globalisation $\left._{(t-1)}\right)$ & & & & $\begin{array}{c}2.164^{* * *} \\
(0.684)\end{array}$ & $\begin{array}{c}2.719 * * * \\
(0.761)\end{array}$ & $\begin{array}{c}3.844^{* * *} \\
(0.963)\end{array}$ & $\begin{array}{c}2.205^{* * *} \\
(0.758)\end{array}$ & $\begin{array}{c}4.516^{* * *} \\
(1.159)\end{array}$ & $\begin{array}{c}4.108^{* * *} \\
(0.898)\end{array}$ \\
\hline $\ln ($ Econ. Globalisation $(t-1))$ & $\begin{array}{c}-1.302^{* * *} \\
(0.370)\end{array}$ & $\begin{array}{c}0.043 \\
(0.699)\end{array}$ & $\begin{array}{c}-1.182^{* * *} \\
(0.416)\end{array}$ & & & & & & \\
\hline $\ln \left(\right.$ Social Globalisation $\left._{(t-1)}\right)$ & $\begin{array}{c}-1.312^{* * * *} \\
(0.353)\end{array}$ & $\begin{array}{l}-0.624 \\
(0.682)\end{array}$ & $\begin{array}{c}-1.738^{* * *} \\
(0.449)\end{array}$ & & & & & & \\
\hline $\ln$ (Pol. Globalisation) & $\begin{array}{l}3.659^{* * *} \\
(0.264)\end{array}$ & $\begin{array}{l}4.174^{* * *} \\
(0.701)\end{array}$ & $\begin{array}{l}3.856^{* * *} \\
(0.303)\end{array}$ & & & & & & \\
\hline $\ln ($ Resource Rents $(t-1))$ & $\begin{array}{c}0.153 \\
(0.100)\end{array}$ & $\begin{array}{c}0.187 \\
(0.121)\end{array}$ & $\begin{array}{l}0.209^{*} \\
(0.124)\end{array}$ & $\begin{array}{c}0.340^{* * *} \\
(0.112)\end{array}$ & $\begin{array}{c}0.349^{* * *} \\
(0.112)\end{array}$ & $\begin{array}{c}0.510^{* * *} \\
(0.147)\end{array}$ & $\begin{array}{c}0.377^{* * *} \\
(0.118)\end{array}$ & $\begin{array}{l}0.302^{* *} \\
(0.128)\end{array}$ & $\begin{array}{l}-0.292 \\
(0.196)\end{array}$ \\
\hline $\ln ($ Primary Educ.(t-1) $)$ & $\begin{array}{c}1.291^{* * *} \\
(0.342)\end{array}$ & $\begin{array}{c}1.505^{* * *} \\
(0.481)\end{array}$ & $\begin{array}{l}1.027^{* *} \\
(0.445)\end{array}$ & $\begin{array}{l}0.918^{* *} \\
(0.365)\end{array}$ & $\begin{array}{c}1.975^{* * *} \\
(0.397)\end{array}$ & $\begin{array}{l}-0.389 \\
(0.566)\end{array}$ & $\begin{array}{l}-0.516 \\
(0.465)\end{array}$ & $\begin{array}{c}-0.125 \\
(0.566)\end{array}$ & $\begin{array}{c}-2.675^{* * *} \\
(0.566)\end{array}$ \\
\hline $\operatorname{Ln}($ Pop. Density $(t-1))$ & $\begin{array}{l}0.168^{* *} \\
(0.068)\end{array}$ & $\begin{array}{c}-0.659^{* * *} \\
(0.128)\end{array}$ & $\begin{array}{l}0.178^{*} \\
(0.095)\end{array}$ & $\begin{array}{l}0.282^{* * *} \\
(0.074)\end{array}$ & $\begin{array}{l}-0.253^{* *} \\
(0.109)\end{array}$ & $\begin{array}{c}0.142 \\
(0.115)\end{array}$ & $\begin{array}{c}0.307^{* * *} \\
(0.097)\end{array}$ & $\begin{array}{c}0.033 \\
(0.134)\end{array}$ & $\begin{array}{l}0.282^{* *} \\
(0.121)\end{array}$ \\
\hline $\ln ($ State Frag. $\cdot(t-1))$ & $\begin{array}{c}1.642^{* * *} \\
(0.280)\end{array}$ & $\begin{array}{c}1.848^{* * *} \\
(0.345)\end{array}$ & $\begin{array}{l}1.366^{* * *} \\
(0.346)\end{array}$ & & & & $\begin{array}{c}1.210^{* * *} \\
(0.365)\end{array}$ & $\begin{array}{l}1.480^{* * *} \\
(0.516)\end{array}$ & $\begin{array}{l}-0.631 \\
(0.569)\end{array}$ \\
\hline $\ln \left(\right.$ Effectiveness $\left._{(t-1)}\right)$ & & & & $\begin{array}{l}0.142 \\
(0.391)\end{array}$ & $\begin{array}{l}1.061^{* *} \\
(0.491)\end{array}$ & $\begin{array}{l}-0.021 \\
(0.490)\end{array}$ & & & \\
\hline $\ln \left(\operatorname{Legitimacy}_{(t-1)}\right)$ & & & & $\begin{array}{c}2.035^{* * *} \\
(0.233)\end{array}$ & $\begin{array}{c}1.225^{* * *} \\
(0.256)\end{array}$ & $\begin{array}{c}2.130^{* * *} \\
(0.378)\end{array}$ & & & \\
\hline $\ln$ (Ruggedness) & & & & & & & $\begin{array}{l}0.177^{* *} \\
(0.071)\end{array}$ & $\begin{array}{l}0.604^{* * *} \\
(0.078)\end{array}$ & $\begin{array}{c}0.189 \\
(0.126)\end{array}$ \\
\hline $\ln ($ Real PCGDP 1950) & & & & & & & $\begin{array}{c}1.075^{* * *} \\
(0.202)\end{array}$ & $\begin{array}{c}1.262^{* * *} \\
(0.336)\end{array}$ & $\begin{array}{l}0.865^{* *} \\
(0.336)\end{array}$ \\
\hline $\ln$ (Dist. Coast) & & & & & & & $\begin{array}{c}0.845^{* * *} \\
(0.163)\end{array}$ & $\begin{array}{c}2.115^{* * * *} \\
(0.296)\end{array}$ & $\begin{array}{c}2.441^{* * * *} \\
(0.187)\end{array}$ \\
\hline $\ln$ (Dist. Atl. Slave) & & & & & & & $\begin{array}{l}0.704^{* *} \\
(0.299)\end{array}$ & $\begin{array}{l}-0.268 \\
(0.641)\end{array}$ & $\begin{array}{l}-0.065 \\
(0.397)\end{array}$ \\
\hline $\ln$ (Dist. Indian Slave) & & & & & & & $\begin{array}{l}0.284 \\
(0.196)\end{array}$ & $\begin{array}{l}-0.766^{* *} \\
(0.330)\end{array}$ & $\begin{array}{l}1.582^{* * *} \\
(0.358)\end{array}$ \\
\hline British Colony & & & & & & & $\begin{array}{c}0.444 \\
(0.351)\end{array}$ & $\begin{array}{c}-3.127^{* *} \\
(1.220)\end{array}$ & $\begin{array}{l}1.089^{* *} \\
(0.428)\end{array}$ \\
\hline France Colony & & & & & & & $\begin{array}{l}-0.109 \\
(0.321)\end{array}$ & $\begin{array}{l}-2.194^{*} \\
(1.280)\end{array}$ & $\begin{array}{l}-0.888 \\
(0.551)\end{array}$ \\
\hline Portugal Colony & & & & & & & $\begin{array}{c}0.017 \\
(0.493)\end{array}$ & $\begin{array}{l}-2.831^{* *} \\
(1.403)\end{array}$ & $\begin{array}{l}4.346^{* * *} \\
(1.262)\end{array}$ \\
\hline lnalpha & $\begin{array}{l}0.824^{* * *} \\
(0.051)\end{array}$ & $\begin{array}{l}0.575^{* * *} \\
(0.106)\end{array}$ & $\begin{array}{l}0.723^{* * *} \\
(0.065)\end{array}$ & $\begin{array}{l}0.995^{* * *} \\
(0.048)\end{array}$ & $\begin{array}{c}0.789^{* * *} \\
(0.087)\end{array}$ & $\begin{array}{c}0.907^{* * *} \\
(0.061)\end{array}$ & $\begin{array}{l}0.825^{* * *} \\
(0.055)\end{array}$ & $\begin{array}{l}0.348^{* * *} \\
(0.095)\end{array}$ & $\begin{array}{l}0.576^{* * *} \\
(0.076)\end{array}$ \\
\hline LogLik & -2424.162 & & -1517.060 & -2497.754 & -893.576 & -1555.749 & -2294.619 & -824.238 & -1363.378 \\
\hline Pseudo-R2 & 0.074 & 0.111 & 0.052 & 0.042 & 0.064 & 0.027 & 0.076 & 0.143 & 0.067 \\
\hline Obs & 658 & 314 & 344 & 645 & 301 & 344 & 623 & 314 & 309 \\
\hline
\end{tabular}


Table 6: Negative Binomial: Civilians/Protests

\begin{tabular}{|c|c|c|c|c|c|c|c|c|c|}
\hline \multirow[b]{2}{*}{ (1) } & \multicolumn{3}{|c|}{ Globalisation } & \multicolumn{3}{|c|}{ Fragility } & \multicolumn{3}{|c|}{ Geographic } \\
\hline & $\begin{array}{l}(2) \\
\text { All }\end{array}$ & $\begin{array}{l}\text { (3) } \\
\mathrm{LQ}\end{array}$ & $\begin{array}{l}\text { (4) } \\
\mathrm{HQ}\end{array}$ & $\begin{array}{l}\text { (5) } \\
\text { All }\end{array}$ & $\begin{array}{l}(6) \\
\mathrm{LQ}\end{array}$ & $\begin{array}{l}(7) \\
\mathrm{HQ}\end{array}$ & $\begin{array}{l}(8) \\
\text { All }\end{array}$ & $\begin{array}{l}\text { (9) } \\
\text { LQ }\end{array}$ & $\begin{array}{l}(10) \\
\text { HQ }\end{array}$ \\
\hline $\ln ($ Military Exp.t-1) & $\begin{array}{l}0.191^{*} \\
(0.110)\end{array}$ & $\begin{array}{c}1.500^{* * *} \\
(0.338)\end{array}$ & $\begin{array}{c}0.018 \\
(0.120)\end{array}$ & $\begin{array}{c}0.121 \\
(0.158)\end{array}$ & $\begin{array}{c}1.210^{* * *} \\
(0.278)\end{array}$ & $\begin{array}{c}0.221 \\
(0.199)\end{array}$ & $\begin{array}{c}0.040 \\
(0.159)\end{array}$ & $\begin{array}{c}1.210^{* * *} \\
(0.351)\end{array}$ & $\begin{array}{c}-0.012 \\
(0.196)\end{array}$ \\
\hline $\ln \left(\right.$ Real $\left.\operatorname{PCGDP}_{(t-1)}\right)$ & $\begin{array}{c}0.044 \\
(0.110)\end{array}$ & $\begin{array}{c}-1.180^{* * *} \\
(0.205)\end{array}$ & $\begin{array}{c}0.422^{* * *} \\
(0.116)\end{array}$ & $\begin{array}{l}-0.136 \\
(0.162)\end{array}$ & $\begin{array}{c}-1.384^{* * *} \\
(0.264)\end{array}$ & $\begin{array}{l}-0.025 \\
(0.179)\end{array}$ & $\begin{array}{c}-0.487^{* * *} \\
(0.138)\end{array}$ & $\begin{array}{c}-1.487^{* * *} \\
(0.197)\end{array}$ & $\begin{array}{c}0.599^{* * *} \\
(0.209)\end{array}$ \\
\hline $\ln \left(\right.$ Globalisation $\left._{(t-1)}\right)$ & & & & $\begin{array}{c}4.743^{* * *} \\
(0.733)\end{array}$ & $\begin{array}{c}3.687^{* * *} \\
(0.941)\end{array}$ & $\begin{array}{c}4.123^{* * *} \\
(0.757)\end{array}$ & $\begin{array}{c}5.818^{* * *} \\
(0.652)\end{array}$ & $\begin{array}{c}5.236^{* * *} \\
(1.048)\end{array}$ & $\begin{array}{c}6.043^{* * *} \\
(0.810)\end{array}$ \\
\hline $\ln ($ Econ. Globalisation $(t-1))$ & $\begin{array}{c}-0.649^{*} \\
(0.393)\end{array}$ & $\begin{array}{c}0.187 \\
(0.531)\end{array}$ & $\begin{array}{c}-1.875^{* * *} \\
(0.412)\end{array}$ & & & & & & \\
\hline $\ln ($ Social Globalisation $(t-1))$ & $\begin{array}{l}0.861^{*} \\
(0.517)\end{array}$ & $\begin{array}{l}-1.158^{*} \\
(0.629)\end{array}$ & $\begin{array}{c}1.483^{* * *} \\
(0.462)\end{array}$ & & & & & & \\
\hline $\ln ($ Pol. Globalisation $)$ & $\begin{array}{c}4.171^{* * *} \\
(0.270)\end{array}$ & $\begin{array}{c}4.993^{* * *} \\
(0.605)\end{array}$ & $\begin{array}{c}3.500^{* * *} \\
(0.281)\end{array}$ & & & & & & \\
\hline $\ln \left(\right.$ Resource $\left.\operatorname{Rents}_{(t-1)}\right)$ & $\begin{array}{c}0.485^{* * *} \\
(0.091)\end{array}$ & $\begin{array}{c}0.352^{* * *} \\
(0.126)\end{array}$ & $\begin{array}{c}0.566^{* * *} \\
(0.095)\end{array}$ & $\begin{array}{c}0.613^{* * *} \\
(0.103)\end{array}$ & $\begin{array}{c}0.629^{* * *} \\
(0.127)\end{array}$ & $\begin{array}{c}0.582^{* * *} \\
(0.113)\end{array}$ & $\begin{array}{c}0.879^{* * *} \\
(0.095)\end{array}$ & $\begin{array}{c}0.430^{* * *} \\
(0.149)\end{array}$ & $\begin{array}{c}0.541^{* * *} \\
(0.129)\end{array}$ \\
\hline $\ln ($ Primary Educ.(t-1) $)$ & $\begin{array}{c}0.641 \\
(0.421)\end{array}$ & $\begin{array}{c}3.389^{* * *} \\
(0.658)\end{array}$ & $\begin{array}{c}0.624 \\
(0.417)\end{array}$ & $\begin{array}{c}0.540 \\
(0.353)\end{array}$ & $\begin{array}{c}3.114^{* * *} \\
(0.677)\end{array}$ & $\begin{array}{l}0.982^{* *} \\
(0.462)\end{array}$ & $\begin{array}{l}-0.514 \\
(0.352)\end{array}$ & $\begin{array}{c}1.026 \\
(0.697)\end{array}$ & $\begin{array}{l}-0.616 \\
(0.490)\end{array}$ \\
\hline $\operatorname{Ln}($ Pop. Density $(t-1))$ & $\begin{array}{c}0.051 \\
(0.065)\end{array}$ & $\begin{array}{c}-0.342^{* *} \\
(0.134)\end{array}$ & $\begin{array}{c}-0.212^{*} \\
(0.129)\end{array}$ & $\begin{array}{c}0.342^{* * *} \\
(0.087)\end{array}$ & $\begin{array}{c}0.087 \\
(0.114)\end{array}$ & $\begin{array}{c}0.217 \\
(0.230)\end{array}$ & $\begin{array}{c}0.238^{* * *} \\
(0.089)\end{array}$ & $\begin{array}{c}0.176 \\
(0.120)\end{array}$ & $\begin{array}{l}-0.096 \\
(0.153)\end{array}$ \\
\hline $\ln ($ State Frag. $\cdot(t-1))$ & $\begin{array}{l}-0.170 \\
(0.278)\end{array}$ & $\begin{array}{c}-1.315^{* *} \\
(0.518)\end{array}$ & $\begin{array}{l}-0.199 \\
(0.301)\end{array}$ & & & & $\begin{array}{c}-0.409 \\
(0.333)\end{array}$ & $\begin{array}{c}-1.088^{* *} \\
(0.466)\end{array}$ & $\begin{array}{c}-0.893 * * \\
(0.392)\end{array}$ \\
\hline $\ln$ Effectiveness $\left._{(t-1)}\right)$ & & & & $\begin{array}{l}-0.570 \\
(0.463)\end{array}$ & $\begin{array}{l}-0.939 \\
(0.603)\end{array}$ & $\begin{array}{c}-1.891^{* * *} \\
(0.529)\end{array}$ & & & \\
\hline $\ln \left(\operatorname{Legitimacy}_{(t-1)}\right)$ & & & & $\begin{array}{c}0.372 \\
(0.385)\end{array}$ & $\begin{array}{c}0.445 \\
(0.358)\end{array}$ & $\begin{array}{c}1.410^{* * *} \\
(0.403)\end{array}$ & & & \\
\hline $\ln$ (Ruggedness) & & & & & & & $\begin{array}{c}0.163^{* * *} \\
(0.055)\end{array}$ & $\begin{array}{c}0.271^{* * *} \\
(0.066)\end{array}$ & $\begin{array}{l}-0.066 \\
(0.077)\end{array}$ \\
\hline ln(Real PCGDP 1950) & & & & & & & $\begin{array}{c}0.881^{* * *} \\
(0.157)\end{array}$ & $\begin{array}{c}1.047^{* * *} \\
(0.205)\end{array}$ & $\begin{array}{c}-0.466^{*} \\
(0.268)\end{array}$ \\
\hline $\ln$ (Dist. Coast) & & & & & & & $\begin{array}{c}0.408^{* * *} \\
(0.153)\end{array}$ & $\begin{array}{c}1.012^{* * *} \\
(0.249)\end{array}$ & $\begin{array}{c}0.830^{* * *} \\
(0.157)\end{array}$ \\
\hline $\ln$ (Dist. Atl. Slave) & & & & & & & $\begin{array}{c}0.987^{* * *} \\
(0.261)\end{array}$ & $\begin{array}{c}1.593 * * * \\
(0.577)\end{array}$ & $\begin{array}{c}0.514 \\
(0.314)\end{array}$ \\
\hline $\ln$ (Dist. Indian Slave) & & & & & & & $\begin{array}{c}0.035 \\
(0.153)\end{array}$ & $\begin{array}{c}0.008 \\
(0.275)\end{array}$ & $\begin{array}{l}-0.348 \\
(0.236)\end{array}$ \\
\hline British Colony & & & & & & & $\begin{array}{c}0.294 \\
(0.369)\end{array}$ & $\begin{array}{c}-1.433^{* * *} \\
(0.538)\end{array}$ & $\begin{array}{c}-1.276^{* *} \\
(0.594)\end{array}$ \\
\hline France Colony & & & & & & & $\begin{array}{c}0.546 \\
(0.343)\end{array}$ & $\begin{array}{l}-0.548 \\
(0.383)\end{array}$ & $\begin{array}{l}-0.948^{*} \\
(0.550)\end{array}$ \\
\hline Portugal Colony & & & & & & & $\begin{array}{l}-0.319 \\
(0.419)\end{array}$ & $\begin{array}{c}-1.738^{* * *} \\
(0.543)\end{array}$ & $\begin{array}{c}0.089 \\
(0.865)\end{array}$ \\
\hline Inalpha & $\begin{array}{c}0.647^{* * *} \\
(0.075)\end{array}$ & $\begin{array}{c}0.466 * * * \\
(0.102)\end{array}$ & $\begin{array}{c}0.441^{* * *} \\
(0.076)\end{array}$ & $\begin{array}{c}0.860^{* * *} \\
(0.068)\end{array}$ & $\begin{array}{c}0.679^{* * *} \\
(0.100)\end{array}$ & $\begin{array}{c}0.744^{* * *} \\
(0.092)\end{array}$ & $\begin{array}{c}0.652^{* * *} \\
(0.066)\end{array}$ & $\begin{array}{c}0.324^{* * *} \\
(0.108)\end{array}$ & $\begin{array}{c}0.509^{* * *} \\
(0.086)\end{array}$ \\
\hline LogLik & -2350.350 & -801.398 & -1474.492 & -2424.321 & -837.640 & -1534.804 & -2236.854 & -785.452 & -1380.998 \\
\hline Pseudo-R2 & 0.093 & 0.126 & 0.104 & 0.060 & 0.075 & 0.067 & 0.093 & 0.144 & 0.090 \\
\hline Obs & 658 & 280 & 378 & 645 & 267 & 378 & 623 & 280 & 343 \\
\hline
\end{tabular}




\section{Appendix A}

Table 1: List of Countries by Type and Quantiles

\begin{tabular}{|c|c|c|c|}
\hline \multicolumn{2}{|c|}{ All Conflict } & \multicolumn{2}{|c|}{ Governments/Related Parties } \\
\hline 1st Quantile & 2nd Quantile & 1st Quantile & 2nd Quantile \\
\hline Algeria & Burundi & Algeria & Angola \\
\hline Angola & Central African Republic & Benin & Burundi \\
\hline Benin & Congo, Dem. Rep. & Botswana & Congo, Dem. Rep. \\
\hline Botswana & Djibouti & Burkina Faso & Djibouti \\
\hline Burkina Faso & Egypt & Cameroon & Egypt \\
\hline Cameroon & Ethiopia & Central African Republic & Equatorial Guinea \\
\hline Chad & Gambia & Chad & Eritrea \\
\hline Equatorial Guinea & Guinea & Gabon & Ethiopia \\
\hline Eritrea & Guinea Bissau & Ghana & Gambia \\
\hline Gabon & Ivory Coast & Madagascar & Guinea \\
\hline Ghana & Kenya & Malawi & Guinea Bissau \\
\hline Lesotho & Liberia & Mali & Ivory Coast \\
\hline Madagascar & Libya & Mauritania & Kenya \\
\hline Mali & Malawi & Morocco & Lesotho \\
\hline Mauritania & Nigeria & Mozambique & Liberia \\
\hline Morocco & Rwanda & Namibia & Libya \\
\hline Mozambique & Senegal & Niger & Nigeria \\
\hline Namibia & Sierra Leone & Republic of Congo & Rwanda \\
\hline Niger & Somalia & South Africa & Senegal \\
\hline Republic of Congo & Somaliland & Togo & Sierra Leone \\
\hline Tanzania & South Africa & Tanzania & Somalia \\
\hline Western Sahara & South Sudan & Western Sahara & Somaliland \\
\hline \multirow[t]{6}{*}{ Zambia } & Sudan & Zambia & South Sudan \\
\hline & Swaziland & & Sudan \\
\hline & Togo & & Swaziland \\
\hline & Tunisia & & Tunisia \\
\hline & Uganda & & Uganda \\
\hline & Zimbabwe & & Zimbabwe \\
\hline
\end{tabular}

\begin{tabular}{|c|c|c|c|}
\hline \multicolumn{2}{|c|}{ Groups/Militias } & \multicolumn{2}{|c|}{ Civilians/Protests } \\
\hline 1st Quantile & 2nd Quantile & 1st Quantile & 2nd Quantile \\
\hline Algeria & Burundi & Algeria & Burkina Faso \\
\hline Angola & Cameroon & Angola & Burundi \\
\hline Benin & Central African Republic & Benin & Djibouti \\
\hline Botswana & Congo, Dem. Rep. & Botswana & Egypt \\
\hline Burkina Faso & Egypt & Cameroon & Ethiopia \\
\hline Chad & Ethiopia & Central African Republic & Gambia \\
\hline Djibouti & Gambia & Chad & Ghana \\
\hline Equatorial Guinea & Guinea & Congo, Dem. Rep. & Guinea \\
\hline Eritrea & Guinea Bissau & Equatorial Guinea & Guinea Bissau \\
\hline Gabon & Ivory Coast & Eritrea & Ivory Coast \\
\hline Ghana & Kenya & Gabon & Kenya \\
\hline Madagascar & Lesotho & Libya & Lesotho \\
\hline Mali & Liberia & Madagascar & Liberia \\
\hline Mauritania & Libya & Mali & Malawi \\
\hline Morocco & Malawi & Mauritania & Morocco \\
\hline Mozambique & Nigeria & Mozambique & Nigeria \\
\hline Namibia & Rwanda & Namibia & Rwanda \\
\hline Niger & Senegal & Niger & Senegal \\
\hline Republic of Congo & Sierra Leone & Republic of Congo & Sierra Leone \\
\hline Togo & Somalia & South Sudan & Somalia \\
\hline Tanzania & Somaliland & Tanzania & Somaliland \\
\hline Western Sahara & South Africa & Zambia & South Africa \\
\hline \multirow[t]{7}{*}{ Zambia } & South Sudan & & Sudan \\
\hline & Sudan & & Swaziland \\
\hline & Swaziland & & Togo \\
\hline & Tunisia & & Tunisia \\
\hline & Uganda & & Uganda \\
\hline & Zimbabwe & & Western Sahara \\
\hline & & & Zimbabwe \\
\hline
\end{tabular}

The first quantile represents countries with $0-50 \%$ occurrences of conflict, while the second quantile represents coun-

tries with $51-100 \%$ occurrences. 


\section{Appendix B}

Table 1: Negative Binomial (Secondary Education)

\begin{tabular}{|c|c|c|c|c|c|c|c|c|c|c|c|c|}
\hline \multirow[b]{2}{*}{ (1) } & \multicolumn{6}{|c|}{ All Conflicts } & \multicolumn{6}{|c|}{ Governments/Related Parties } \\
\hline & $\begin{array}{l}(2) \\
\text { All }\end{array}$ & $\begin{array}{l}\text { (3) } \\
\text { All Sq. }\end{array}$ & $\begin{array}{l}\text { (4) } \\
\text { LQ }\end{array}$ & $\begin{array}{c}(5) \\
\text { LQ Sq. }\end{array}$ & $\begin{array}{l}6) \\
\mathrm{HQ}\end{array}$ & $\begin{array}{l}(7) \\
\text { HQ Sq. }\end{array}$ & $\begin{array}{l}(8) \\
\text { All }\end{array}$ & $\begin{array}{l}(9) \\
\text { All Sq. }\end{array}$ & $\begin{array}{l}(10) \\
\text { LQ }\end{array}$ & $\begin{array}{c}(11) \\
\text { LQ Sq. }\end{array}$ & $\begin{array}{l}12 \\
\mathrm{HQ}\end{array}$ & $\begin{array}{l}(13) \\
\text { HQ Sq. }\end{array}$ \\
\hline $\ln ($ Military Exp.t-1) & $\begin{array}{l}0.574^{* * *} \\
(0.151)\end{array}$ & $\begin{array}{c}0.634^{* * *} \\
(0.208)\end{array}$ & $\begin{array}{c}0.656^{* * *} \\
(0.164)\end{array}$ & $\begin{array}{l}-0.025 \\
(0.308)\end{array}$ & $\begin{array}{c}0.577^{* * *} \\
(0.217)\end{array}$ & $\begin{array}{l}-0.097 \\
(0.307)\end{array}$ & $\begin{array}{c}0.651^{* * *} \\
(0.172)\end{array}$ & $\begin{array}{c}0.807^{* * * *} \\
(0.258)\end{array}$ & $\begin{array}{l}0.546^{* *} \\
(0.255)\end{array}$ & $\begin{array}{c}1.251^{* * *} \\
(0.342)\end{array}$ & $\begin{array}{c}0.333 \\
(0.242)\end{array}$ & $\begin{array}{l}-0.198 \\
(0.382)\end{array}$ \\
\hline $\ln \left(\right.$ Military $\left.\operatorname{Exp}_{\cdot t-1}\right)$ Sq. & & $\begin{array}{l}-0.194^{*} \\
(0.111)\end{array}$ & & $\begin{array}{l}-0.112 \\
(0.113)\end{array}$ & & $\begin{array}{c}0.233 \\
(0.159)\end{array}$ & & $\begin{array}{l}-0.220^{*} \\
(0.130)\end{array}$ & & $\begin{array}{l}-0.493^{*} \\
(0.253)\end{array}$ & & $\begin{array}{c}0.226 \\
(0.146)\end{array}$ \\
\hline $\ln \left(\right.$ Real $\left.\operatorname{PCGDP}_{(t-1)}\right)$ & $\begin{array}{c}0.052 \\
(0.162)\end{array}$ & $\begin{array}{c}-4.112^{* * *} \\
(1.545)\end{array}$ & $\begin{array}{l}-0.220 \\
(0.308)\end{array}$ & $\begin{array}{c}5.804 \\
(5.258)\end{array}$ & $\begin{array}{c}0.008 \\
(0.205)\end{array}$ & $\begin{array}{l}-1.802 \\
(1.965)\end{array}$ & $\begin{array}{c}0.250 \\
(0.213)\end{array}$ & $\begin{array}{l}-3.681^{*} \\
(2.109)\end{array}$ & $\begin{array}{c}0.193 \\
(0.280)\end{array}$ & $\begin{array}{l}-5.045^{*} \\
(3.008)\end{array}$ & $\begin{array}{l}-0.044 \\
(0.214)\end{array}$ & $\begin{array}{c}-6.654^{* *} \\
(3.159)\end{array}$ \\
\hline $\ln \left(\right.$ Real PCGDP $\left._{(t-1)}\right)$ Sq. & & $\begin{array}{l}0.281^{* *} \\
(0.112)\end{array}$ & & $\begin{array}{l}-0.392 \\
(0.378)\end{array}$ & & $\begin{array}{c}0.103 \\
(0.141)\end{array}$ & & $\begin{array}{l}0.274^{*} \\
(0.156)\end{array}$ & & $\begin{array}{c}0.301 \\
(0.221)\end{array}$ & & $\begin{array}{l}0.476^{* *} \\
(0.232)\end{array}$ \\
\hline $\ln \left(\right.$ Globalisation $\left._{(t-1)}\right)$ & $\begin{array}{l}2.634^{* * *} \\
(0.725)\end{array}$ & $\begin{array}{c}-49.256^{* * *} \\
(10.810)\end{array}$ & $\begin{array}{l}4.307^{* * *} \\
(0.756)\end{array}$ & $\begin{array}{c}-62.913^{* * *} \\
(13.163)\end{array}$ & $\begin{array}{l}2.538^{* * *} \\
(0.973)\end{array}$ & $\begin{array}{c}-45.279^{* * * *} \\
(14.026)\end{array}$ & $\begin{array}{c}1.171 \\
(0.784)\end{array}$ & $\begin{array}{c}-38.948^{* * *} \\
(14.346)\end{array}$ & $\begin{array}{l}-0.801 \\
(1.253)\end{array}$ & $\begin{array}{c}-124.633^{* * *} \\
(25.242)\end{array}$ & $\begin{array}{l}2.171^{* *} \\
(0.923)\end{array}$ & $\begin{array}{c}4.674 \\
(18.048)\end{array}$ \\
\hline $\ln \left(\right.$ Globalisation $\left._{(t-1)}\right)$ Sq. & & $\begin{array}{c}7.122^{* * *} \\
(1.426)\end{array}$ & & $\begin{array}{c}8.845^{* * *} \\
(1.764)\end{array}$ & & $\begin{array}{c}6.538^{* * *} \\
(1.860)\end{array}$ & & $\begin{array}{l}5.495^{* * *} \\
(1.896)\end{array}$ & & $\begin{array}{c}15.991^{* * *} \\
(3.292)\end{array}$ & & $\begin{array}{l}-0.088 \\
(2.453)\end{array}$ \\
\hline $\ln \left(\operatorname{Resource}^{\left.\operatorname{Rents}_{(t-1)}\right)}\right.$ & $\begin{array}{l}0.809^{* * *} \\
(0.111)\end{array}$ & $\begin{array}{c}0.686^{* * *} \\
(0.173)\end{array}$ & $\begin{array}{c}0.497^{* * *} \\
(0.132)\end{array}$ & $\begin{array}{l}-0.084 \\
(0.217)\end{array}$ & $\begin{array}{c}1.074^{* * *} \\
(0.142)\end{array}$ & $\begin{array}{c}1.595^{* * *} \\
(0.176)\end{array}$ & $\begin{array}{l}0.754^{* * *} \\
(0.129)\end{array}$ & $\begin{array}{l}0.402^{* *} \\
(0.163)\end{array}$ & $\begin{array}{c}0.659^{* * *} \\
(0.187)\end{array}$ & $\begin{array}{c}-0.608^{* *} \\
(0.269)\end{array}$ & $\begin{array}{c}1.031^{* * *} \\
(0.130)\end{array}$ & $\begin{array}{c}1.447^{* * * *} \\
(0.226)\end{array}$ \\
\hline $\ln \left(\operatorname{Resource} \operatorname{Rents}_{(t-1)}\right)$ Sq. & & $\begin{array}{c}-0.111^{* *} \\
(0.047)\end{array}$ & & $\begin{array}{c}0.182^{* * *} \\
(0.064)\end{array}$ & & $\begin{array}{c}-0.357^{* * *} \\
(0.051)\end{array}$ & & $\begin{array}{l}-0.023 \\
(0.048)\end{array}$ & & $\begin{array}{c}0.278^{* * *} \\
(0.074)\end{array}$ & & $\begin{array}{c}-0.365^{* * *} \\
(0.072)\end{array}$ \\
\hline $\ln ($ Secondary Educ. $(t-1))$ & $\begin{array}{l}0.911^{* * *} \\
(0.215)\end{array}$ & $\begin{array}{c}0.887 \\
(1.644)\end{array}$ & $\begin{array}{c}0.339 \\
(0.409)\end{array}$ & $\begin{array}{l}-4.026 \\
(2.743)\end{array}$ & $\begin{array}{c}0.799^{* * *} \\
(0.255)\end{array}$ & $\begin{array}{l}-0.858 \\
(2.783)\end{array}$ & $\begin{array}{l}0.694^{* *} \\
(0.303)\end{array}$ & $\begin{array}{l}-0.305 \\
(2.491)\end{array}$ & $\begin{array}{l}0.829^{* *} \\
(0.363)\end{array}$ & $\begin{array}{c}1.714 \\
(1.940)\end{array}$ & $\begin{array}{c}0.427 \\
(0.298)\end{array}$ & $\begin{array}{c}-9.534^{* * *} \\
(3.671)\end{array}$ \\
\hline $\ln \left(\right.$ Secondary Educ. $\left.{ }_{(t-1)}\right)$ Sq. & & $\begin{array}{l}-0.007 \\
(0.257)\end{array}$ & & $\begin{array}{l}0.607 \\
(0.434)\end{array}$ & & $\begin{array}{c}0.266 \\
(0.418)\end{array}$ & & $\begin{array}{c}0.134 \\
(0.378)\end{array}$ & & $\begin{array}{c}0.035 \\
(0.318)\end{array}$ & & $\begin{array}{c}1.425^{* * *} \\
(0.546)\end{array}$ \\
\hline $\operatorname{Ln}($ Pop. Density $(t-1))$ & $\begin{array}{l}0.204^{* *} \\
(0.085)\end{array}$ & $\begin{array}{l}0.607^{*} \\
(0.348)\end{array}$ & $\begin{array}{l}-0.055 \\
(0.109)\end{array}$ & $\begin{array}{l}4.362^{* * *} \\
(0.787)\end{array}$ & $\begin{array}{l}-0.102 \\
(0.125)\end{array}$ & $\begin{array}{c}0.347 \\
(0.702)\end{array}$ & $\begin{array}{c}0.175 \\
(0.108)\end{array}$ & $\begin{array}{c}0.675 \\
(0.450)\end{array}$ & $\begin{array}{l}-0.125 \\
(0.157)\end{array}$ & $\begin{array}{l}1.995^{* * *} \\
(0.526)\end{array}$ & $\begin{array}{l}-0.159 \\
(0.142)\end{array}$ & $\begin{array}{c}1.297 \\
(1.183)\end{array}$ \\
\hline Ln(Pop. Density $(t-1)$ Sq. & & $\begin{array}{l}-0.083^{*} \\
(0.048)\end{array}$ & & $\begin{array}{c}-0.832^{* * *} \\
(0.155)\end{array}$ & & $\begin{array}{l}-0.062 \\
(0.089)\end{array}$ & & $\begin{array}{l}-0.080 \\
(0.057)\end{array}$ & & $\begin{array}{c}-0.337^{* * *} \\
(0.092)\end{array}$ & & $\begin{array}{l}-0.173 \\
(0.131)\end{array}$ \\
\hline $\ln ($ State Frag. $(t-1))$ & $\begin{array}{l}1.460^{* * *} \\
(0.406)\end{array}$ & $\begin{array}{c}5.581^{* * *} \\
(0.745)\end{array}$ & $\begin{array}{l}1.919^{* * *} \\
(0.499)\end{array}$ & $\begin{array}{c}-3.967^{* * *} \\
(1.538)\end{array}$ & $\begin{array}{l}0.764^{*} \\
(0.443)\end{array}$ & $\begin{array}{c}7.395^{* * *} \\
(1.071)\end{array}$ & $\begin{array}{l}1.825^{* * *} \\
(0.425)\end{array}$ & $\begin{array}{c}5.461^{* * *} \\
(0.927)\end{array}$ & $\begin{array}{c}0.131 \\
(0.802)\end{array}$ & $\begin{array}{c}-4.008^{* * *} \\
(1.300)\end{array}$ & $\begin{array}{l}1.221^{* *} \\
(0.477)\end{array}$ & $\begin{array}{c}9.177^{* * * *} \\
(1.217)\end{array}$ \\
\hline $\ln ($ State Frag. $(t-1))$ Sq. & & $\begin{array}{c}1.379^{* * *} \\
(0.378)\end{array}$ & & $\begin{array}{c}-2.576^{* * *} \\
(0.608)\end{array}$ & & $\begin{array}{c}2.830^{* * *} \\
(0.475)\end{array}$ & & $\begin{array}{c}1.160^{* * *} \\
(0.407)\end{array}$ & & $\begin{array}{c}-3.110^{* * *} \\
(0.678)\end{array}$ & & $\begin{array}{c}2.994^{* * *} \\
(0.505)\end{array}$ \\
\hline lnalpha & $\begin{array}{c}0.666^{* * *} \\
(0.052)\end{array}$ & $\begin{array}{l}0.516^{* * *} \\
(0.061)\end{array}$ & $\begin{array}{l}0.484^{* * *} \\
(0.095)\end{array}$ & $\begin{array}{c}0.254^{* * *} \\
(0.096)\end{array}$ & $\begin{array}{l}0.555^{* * *} \\
(0.071)\end{array}$ & $\begin{array}{l}0.312^{* * *} \\
(0.086)\end{array}$ & $\begin{array}{l}1.086^{* * *} \\
(0.071)\end{array}$ & $\begin{array}{c}1.007^{* * *} \\
(0.075)\end{array}$ & $\begin{array}{c}0.922^{* * *} \\
(0.111)\end{array}$ & $\begin{array}{c}0.567^{* * *} \\
(0.120)\end{array}$ & $\begin{array}{c}0.910^{* * *} \\
(0.087)\end{array}$ & $\begin{array}{c}0.705^{* * *} \\
(0.098)\end{array}$ \\
\hline LogLik & -2361.731 & -2315.732 & -999.448 & -966.425 & -1316.125 & -1278.125 & -1586.806 & -1568.586 & -626.944 & -589.255 & -922.245 & -897.382 \\
\hline Pseudo-R2 & 0.058 & 0.077 & 0.088 & 0.118 & 0.033 & 0.061 & 0.060 & 0.071 & 0.083 & 0.138 & 0.040 & 0.066 \\
\hline Obs & 490 & 490 & 259 & 259 & 231 & 231 & 490 & 490 & 264 & 264 & 226 & 226 \\
\hline
\end{tabular}

Robust Standard errors in parentheses. " $p<.10,{ }^{* *} p<.05,{ }^{* * *} p<.01$. Note that the headers "All", "LQ", and "HQ" indicate that all conflicts of that type were used, only the lower quantile was used, and only

the upper quatile was used in the regressions. 
Table 2: Negative Binomial (Secondary Education)

\begin{tabular}{|c|c|c|c|c|c|c|c|c|c|c|c|c|}
\hline \multirow[b]{2}{*}{ (1) } & \multicolumn{6}{|c|}{ Groups/Militias } & \multicolumn{6}{|c|}{ Civilians/Protests } \\
\hline & $\begin{array}{l}(2) \\
\text { All }\end{array}$ & $\begin{array}{c}(3) \\
\text { All Sq. }\end{array}$ & $\begin{array}{l}\text { (4) } \\
\text { LQ }\end{array}$ & $\begin{array}{c}(5) \\
\text { LQ Sq. }\end{array}$ & $\begin{array}{l}(6) \\
\mathrm{HQ}\end{array}$ & $\begin{array}{c}(7) \\
\text { HQ Sq. }\end{array}$ & $\begin{array}{l}(8) \\
\text { All }\end{array}$ & $\begin{array}{c}(9) \\
\text { All Sq. }\end{array}$ & $\begin{array}{l}(10) \\
\text { LQ }\end{array}$ & $\begin{array}{c}\text { (11) } \\
\text { LQ Sq. }\end{array}$ & $\begin{array}{l}12 \\
\mathrm{HQ}\end{array}$ & $\begin{array}{l}\text { (13) } \\
\text { HQ Sq. }\end{array}$ \\
\hline $\ln \left(\right.$ Military $\left.\operatorname{Exp}_{\cdot t-1}\right)$ & $\begin{array}{c}0.431^{* * *} \\
(0.153)\end{array}$ & $\begin{array}{l}0.363^{*} \\
(0.204)\end{array}$ & $\begin{array}{l}-0.005 \\
(0.159)\end{array}$ & $\begin{array}{c}-1.048^{* * *} \\
(0.298)\end{array}$ & $\begin{array}{c}0.853^{* * *} \\
(0.230)\end{array}$ & $\begin{array}{l}-0.214 \\
(0.307)\end{array}$ & $\begin{array}{c}0.258 \\
(0.187)\end{array}$ & $\begin{array}{c}0.182 \\
(0.279)\end{array}$ & $\begin{array}{c}0.586^{* * *} \\
(0.227)\end{array}$ & $\begin{array}{l}1.281^{* *} \\
(0.519)\end{array}$ & $\begin{array}{l}-0.069 \\
(0.322)\end{array}$ & $\begin{array}{l}-0.156 \\
(0.350)\end{array}$ \\
\hline $\ln \left(\right.$ Military $\left.\operatorname{Exp}_{t-1}\right)$ Sq. & & $\begin{array}{l}-0.124 \\
(0.112)\end{array}$ & & $\begin{array}{c}0.184 \\
(0.123)\end{array}$ & & $\begin{array}{l}0.429^{*} \\
(0.235)\end{array}$ & & $\begin{array}{l}-0.052 \\
(0.107)\end{array}$ & & $\begin{array}{l}-0.307^{*} \\
(0.164)\end{array}$ & & $\begin{array}{l}-0.096 \\
(0.146)\end{array}$ \\
\hline $\ln \left(\right.$ Real $\left.\operatorname{PCGDP}_{(t-1)}\right)$ & $\begin{array}{l}-0.139 \\
(0.151)\end{array}$ & $\begin{array}{l}-2.332 \\
(1.631)\end{array}$ & $\begin{array}{l}-0.494^{* *} \\
(0.251)\end{array}$ & $\begin{array}{l}8.794^{* *} \\
(4.317)\end{array}$ & $\begin{array}{c}0.007 \\
(0.216)\end{array}$ & $\begin{array}{l}-2.300 \\
(2.150)\end{array}$ & $\begin{array}{l}-0.364^{* *} \\
(0.157)\end{array}$ & $\begin{array}{c}-4.870^{* * *} \\
(1.752)\end{array}$ & $\begin{array}{c}-1.232^{* * *} \\
(0.223)\end{array}$ & $\begin{array}{l}-5.431^{*} \\
(3.048)\end{array}$ & $\begin{array}{l}-0.236 \\
(0.237)\end{array}$ & $\begin{array}{c}-4.197^{* *} \\
(2.119)\end{array}$ \\
\hline $\ln ($ Real PCGDP $(t-1))$ Sq. & & $\begin{array}{c}0.131 \\
(0.117)\end{array}$ & & $\begin{array}{c}-0.640^{* *} \\
(0.307)\end{array}$ & & $\begin{array}{c}0.124 \\
(0.153)\end{array}$ & & $\begin{array}{l}0.305^{* *} \\
(0.124)\end{array}$ & & $\begin{array}{c}0.286 \\
(0.220)\end{array}$ & & $\begin{array}{c}0.263^{*} \\
(0.149)\end{array}$ \\
\hline $\ln \left(\right.$ Globalisation $\left._{(t-1)}\right)$ & $\begin{array}{c}2.261^{* * *} \\
(0.846)\end{array}$ & $\begin{array}{c}-49.472^{* * * *} \\
(13.428)\end{array}$ & $\begin{array}{l}2.435^{* * *} \\
(0.726)\end{array}$ & $\begin{array}{c}-36.971^{* *} \\
(15.213)\end{array}$ & $\begin{array}{c}3.732^{* * * *} \\
(1.116)\end{array}$ & $\begin{array}{c}-47.554^{* * *} \\
(15.986)\end{array}$ & $\begin{array}{l}5.596^{* * *} \\
(0.785)\end{array}$ & $\begin{array}{c}-31.812^{* *} \\
(12.436)\end{array}$ & $\begin{array}{l}5.251^{* * *} \\
(1.195)\end{array}$ & $\begin{array}{l}-22.205 \\
(23.178)\end{array}$ & $\begin{array}{l}3.645^{* * *} \\
(1.026)\end{array}$ & $\begin{array}{l}-1.309 \\
(18.409)\end{array}$ \\
\hline $\ln \left(\right.$ Globalisation $\left._{(t-1)}\right)$ Sq. & & $\begin{array}{c}7.190^{* * *} \\
(1.760)\end{array}$ & & $\begin{array}{c}5.294^{* * *} \\
(2.035)\end{array}$ & & $\begin{array}{c}7.147^{* * *} \\
(2.102)\end{array}$ & & $\begin{array}{c}4.996^{* * *} \\
(1.619)\end{array}$ & & $\begin{array}{c}3.644 \\
(3.145)\end{array}$ & & $\begin{array}{c}0.873 \\
(2.395)\end{array}$ \\
\hline $\ln \left(\right.$ Resource $\left.\operatorname{Rents}_{(t-1)}\right)$ & $\begin{array}{l}0.496^{* * *} \\
(0.121)\end{array}$ & $\begin{array}{c}0.487^{* * *} \\
(0.186)\end{array}$ & $\begin{array}{l}0.489^{* * *} \\
(0.121)\end{array}$ & $\begin{array}{l}-0.138 \\
(0.276)\end{array}$ & $\begin{array}{c}0.769^{* * *} \\
(0.179)\end{array}$ & $\begin{array}{c}0.359 \\
(0.734)\end{array}$ & $\begin{array}{c}0.811^{* * *} \\
(0.096)\end{array}$ & $\begin{array}{c}0.916^{* * *} \\
(0.166)\end{array}$ & $\begin{array}{c}0.577^{* * *} \\
(0.130)\end{array}$ & $\begin{array}{c}0.713^{* * *} \\
(0.246)\end{array}$ & $\begin{array}{c}0.769^{* * *} \\
(0.109)\end{array}$ & $\begin{array}{c}0.355 \\
(0.241)\end{array}$ \\
\hline $\ln \left(\right.$ Resource Rents $\left._{(t-1)}\right) \mathrm{Sq}$. & & $\begin{array}{c}-0.123^{* *} \\
(0.050)\end{array}$ & & $\begin{array}{l}0.168^{* *} \\
(0.083)\end{array}$ & & $\begin{array}{l}-0.183 \\
(0.152)\end{array}$ & & $\begin{array}{c}-0.153^{* * *} \\
(0.045)\end{array}$ & & $\begin{array}{l}-0.053 \\
(0.059)\end{array}$ & & $\begin{array}{l}-0.036 \\
(0.067)\end{array}$ \\
\hline $\ln \left(\right.$ Secondary Educ. $\left.{ }_{(t-1)}\right)$ & $\begin{array}{l}1.184^{* * *} \\
(0.204)\end{array}$ & $\begin{array}{c}0.495 \\
(1.995)\end{array}$ & $\begin{array}{l}1.480^{* * *} \\
(0.270)\end{array}$ & $\begin{array}{l}-3.219^{*} \\
(1.711)\end{array}$ & $\begin{array}{l}0.551^{* *} \\
(0.266)\end{array}$ & $\begin{array}{l}-2.518 \\
(3.042)\end{array}$ & $\begin{array}{l}1.039^{* * *} \\
(0.257)\end{array}$ & $\begin{array}{c}0.792 \\
(1.455)\end{array}$ & $\begin{array}{c}1.324^{* * *} \\
(0.276)\end{array}$ & $\begin{array}{l}-1.267 \\
(1.533)\end{array}$ & $\begin{array}{c}1.506^{* * *} \\
(0.378)\end{array}$ & $\begin{array}{l}-2.414 \\
(3.152)\end{array}$ \\
\hline $\ln \left(\right.$ Secondary Educ. $\left._{(t-1)}\right)$ Sq. & & $\begin{array}{c}0.112 \\
(0.295)\end{array}$ & & $\begin{array}{l}0.705^{* *} \\
(0.282)\end{array}$ & & $\begin{array}{c}0.489 \\
(0.451)\end{array}$ & & $\begin{array}{c}0.061 \\
(0.227)\end{array}$ & & $\begin{array}{c}0.401 \\
(0.255)\end{array}$ & & $\begin{array}{c}0.600 \\
(0.473)\end{array}$ \\
\hline $\operatorname{Ln}($ Pop. Density $(t-1))$ & $\begin{array}{l}0.170^{*} \\
(0.094)\end{array}$ & $\begin{array}{l}-0.055 \\
(0.426)\end{array}$ & $\begin{array}{c}-0.418^{* * *} \\
(0.127)\end{array}$ & $\begin{array}{c}3.882^{* * * *} \\
(0.778)\end{array}$ & $\begin{array}{c}0.004 \\
(0.141)\end{array}$ & $\begin{array}{l}-0.620 \\
(0.766)\end{array}$ & $\begin{array}{c}0.293^{* * *} \\
(0.086)\end{array}$ & $\begin{array}{c}0.959^{* * *} \\
(0.283)\end{array}$ & $\begin{array}{l}-0.025 \\
(0.134)\end{array}$ & $\begin{array}{c}0.916 \\
(0.787)\end{array}$ & $\begin{array}{l}-0.047 \\
(0.213)\end{array}$ & $\begin{array}{c}0.001 \\
(2.203)\end{array}$ \\
\hline $\operatorname{Ln}$ Pop. Density $(t-1)$ Sq. & & $\begin{array}{l}-0.003 \\
(0.058)\end{array}$ & & $\begin{array}{c}-0.829^{* * *} \\
(0.145)\end{array}$ & & $\begin{array}{c}0.062 \\
(0.100)\end{array}$ & & $\begin{array}{c}-0.116^{* *} \\
(0.046)\end{array}$ & & $\begin{array}{l}-0.140 \\
(0.163)\end{array}$ & & $\begin{array}{l}-0.016 \\
(0.251)\end{array}$ \\
\hline $\ln \left(\right.$ State Frag. $\left.{ }_{(t-1)}\right)$ & $\begin{array}{c}2.800^{* * *} \\
(0.379)\end{array}$ & $\begin{array}{c}7.439^{* * *} \\
(0.767)\end{array}$ & $\begin{array}{c}2.606^{* * *} \\
(0.399)\end{array}$ & $\begin{array}{l}-0.814 \\
(1.378)\end{array}$ & $\begin{array}{c}2.648^{* * * *} \\
(0.483)\end{array}$ & $\begin{array}{c}10.901^{* * *} \\
(1.081)\end{array}$ & $\begin{array}{c}0.498 \\
(0.387)\end{array}$ & $\begin{array}{c}2.450^{* * *} \\
(0.893)\end{array}$ & $\begin{array}{l}1.036^{* *} \\
(0.517)\end{array}$ & $\begin{array}{l}-1.198 \\
(1.160)\end{array}$ & $\begin{array}{c}0.078 \\
(0.448)\end{array}$ & $\begin{array}{c}5.024^{* * *} \\
(1.630)\end{array}$ \\
\hline $\ln \left(\right.$ State Frag. $\left.{ }_{(t-1)}\right)$ Sq. & & $\begin{array}{c}1.705^{* * *} \\
(0.355)\end{array}$ & & $\begin{array}{c}-1.279^{* *} \\
(0.554)\end{array}$ & & $\begin{array}{c}3.601^{* * *} \\
(0.462)\end{array}$ & & $\begin{array}{c}0.545 \\
(0.457)\end{array}$ & & $\begin{array}{c}-1.286^{* * *} \\
(0.399)\end{array}$ & & $\begin{array}{c}1.970^{* * *} \\
(0.724)\end{array}$ \\
\hline lnalpha & $\begin{array}{c}0.991^{* * *} \\
(0.062)\end{array}$ & $\begin{array}{c}0.862^{* * *} \\
(0.064)\end{array}$ & $\begin{array}{c}0.733^{* * *} \\
(0.117)\end{array}$ & $\begin{array}{c}0.495^{* * *} \\
(0.122)\end{array}$ & $\begin{array}{c}0.930^{* * *} \\
(0.072)\end{array}$ & $\begin{array}{c}0.686^{* * *} \\
(0.081)\end{array}$ & $\begin{array}{c}0.780^{* * *} \\
(0.078)\end{array}$ & $\begin{array}{c}0.647^{* * *} \\
(0.086)\end{array}$ & $\begin{array}{c}0.458^{* * *} \\
(0.127)\end{array}$ & $\begin{array}{l}0.288^{* *} \\
(0.133)\end{array}$ & $\begin{array}{c}0.743^{* * *} \\
(0.097)\end{array}$ & $\begin{array}{c}0.653^{* * *} \\
(0.099)\end{array}$ \\
\hline LogLik & -1811.422 & -1778.157 & -634.736 & -615.682 & -1141.782 & -1104.861 & -1723.033 & -1687.989 & -537.800 & -522.974 & -1148.802 & -1135.038 \\
\hline Pseudo-R2 & 0.062 & 0.079 & 0.109 & 0.136 & 0.032 & 0.063 & 0.090 & 0.109 & 0.132 & 0.156 & 0.074 & 0.085 \\
\hline Obs & 490 & 490 & 236 & 236 & 254 & 254 & 490 & 490 & 206 & 206 & 284 & 284 \\
\hline
\end{tabular}

the upper quatile was used in the regressions. 
Table 3: Negative Binomial: All Conflict (Secondary Education)

\begin{tabular}{|c|c|c|c|c|c|c|c|c|c|}
\hline \multirow[b]{2}{*}{ (1) } & \multicolumn{3}{|c|}{ Globalisation } & \multicolumn{3}{|c|}{ Fragility } & \multicolumn{3}{|c|}{ Geographic } \\
\hline & $\begin{array}{l}\text { (2) } \\
\text { All }\end{array}$ & $\begin{array}{l}\text { (3) } \\
\text { LQ }\end{array}$ & $\begin{array}{l}(4) \\
\text { HQ }\end{array}$ & $\begin{array}{l}\text { (5) } \\
\text { All }\end{array}$ & $\begin{array}{l}\text { (6) } \\
\text { LQ }\end{array}$ & $\begin{array}{l}\text { (7) } \\
\text { HQ }\end{array}$ & $\begin{array}{l}\text { (8) } \\
\text { All }\end{array}$ & $\begin{array}{l}\text { (9) } \\
\text { LQ }\end{array}$ & $\begin{array}{l}(10) \\
\text { HQ }\end{array}$ \\
\hline $\ln ($ Military Exp. $t-1)$ & $\begin{array}{c}0.648^{* * *} \\
(0.118)\end{array}$ & $\begin{array}{l}0.491^{* *} \\
(0.203)\end{array}$ & $\begin{array}{c}0.477^{* * *} \\
(0.135)\end{array}$ & $\begin{array}{c}0.515^{* * *} \\
(0.151)\end{array}$ & $\begin{array}{c}0.623^{* * *} \\
(0.168)\end{array}$ & $\begin{array}{c}0.593^{* * *} \\
(0.207)\end{array}$ & $\begin{array}{c}0.406^{* * *} \\
(0.155)\end{array}$ & $\begin{array}{c}0.116 \\
(0.242)\end{array}$ & $\begin{array}{c}0.842^{* * * *} \\
(0.229)\end{array}$ \\
\hline $\ln \left(\right.$ Real $\left.\operatorname{PCGDP}_{(t-1)}\right)$ & $\begin{array}{l}0.365^{* *} \\
(0.151)\end{array}$ & $\begin{array}{c}-0.461^{*} \\
(0.241)\end{array}$ & $\begin{array}{c}0.472^{* * *} \\
(0.155)\end{array}$ & $\begin{array}{l}-0.152 \\
(0.186)\end{array}$ & $\begin{array}{l}-0.074 \\
(0.346)\end{array}$ & $\begin{array}{l}-0.128 \\
(0.208)\end{array}$ & $\begin{array}{c}-0.557^{* *} \\
(0.271)\end{array}$ & $\begin{array}{l}-0.376 \\
(0.294)\end{array}$ & $\begin{array}{l}-0.126 \\
(0.478)\end{array}$ \\
\hline $\ln \left(\right.$ Globalisation $\left._{(t-1)}\right)$ & & & & $\begin{array}{c}2.373^{* * *} \\
(0.729)\end{array}$ & $\begin{array}{c}4.251^{* * *} \\
(0.790)\end{array}$ & $\begin{array}{c}2.552^{* * * *} \\
(0.905)\end{array}$ & $\begin{array}{l}1.679^{* *} \\
(0.831)\end{array}$ & $\begin{array}{c}4.893^{* * *} \\
(1.019)\end{array}$ & $\begin{array}{l}2.860^{* *} \\
(1.250)\end{array}$ \\
\hline $\ln ($ Econ. Globalisation $(t-1))$ & $\begin{array}{c}-0.959^{* *} \\
(0.390)\end{array}$ & $\begin{array}{c}2.821^{* * *} \\
(0.812)\end{array}$ & $\begin{array}{c}-2.083^{* * *} \\
(0.398)\end{array}$ & & & & & & \\
\hline $\ln \left(\right.$ Social Globalisation $\left._{(t-1)}\right)$ & $\begin{array}{c}-1.173^{* * *} \\
(0.426)\end{array}$ & $\begin{array}{c}-2.730^{* * * *} \\
(0.652)\end{array}$ & $\begin{array}{l}-0.239 \\
(0.498)\end{array}$ & & & & & & \\
\hline $\ln$ (Pol. Globalisation) & $\begin{array}{c}3.427^{* * *} \\
(0.276)\end{array}$ & $\begin{array}{c}4.920^{* * *} \\
(0.578)\end{array}$ & $\begin{array}{c}3.408^{* * *} \\
(0.319)\end{array}$ & & & & & & \\
\hline $\ln ($ Resource Rents $(t-1))$ & $\begin{array}{c}0.561^{* * *} \\
(0.090)\end{array}$ & $\begin{array}{c}0.130 \\
(0.136)\end{array}$ & $\begin{array}{l}0.676^{* * *} \\
(0.124)\end{array}$ & $\begin{array}{c}0.715^{* * *} \\
(0.120)\end{array}$ & $\begin{array}{c}0.554^{* * *} \\
(0.138)\end{array}$ & $\begin{array}{c}1.037^{* * *} \\
(0.144)\end{array}$ & $\begin{array}{c}0.781^{* * *} \\
(0.110)\end{array}$ & $\begin{array}{c}0.537^{* * *} \\
(0.119)\end{array}$ & $\begin{array}{c}0.396^{*} \\
(0.231)\end{array}$ \\
\hline $\ln \left(\right.$ Secondary Educ. $\left.{ }_{(t-1)}\right)$ & $\begin{array}{c}0.648^{* * *} \\
(0.190)\end{array}$ & $\begin{array}{c}1.480^{* * *} \\
(0.305)\end{array}$ & $\begin{array}{c}0.154 \\
(0.295)\end{array}$ & $\begin{array}{c}0.875^{* * *} \\
(0.223)\end{array}$ & $\begin{array}{c}0.422 \\
(0.394)\end{array}$ & $\begin{array}{c}0.784^{* * *} \\
(0.264)\end{array}$ & $\begin{array}{l}0.749^{* *} \\
(0.311)\end{array}$ & $\begin{array}{c}0.243 \\
(0.311)\end{array}$ & $\begin{array}{c}0.417 \\
(0.508)\end{array}$ \\
\hline $\operatorname{Ln}($ Pop. Density $(t-1))$ & $\begin{array}{c}0.118 \\
(0.073)\end{array}$ & $\begin{array}{c}-0.331^{* * *} \\
(0.099)\end{array}$ & $\begin{array}{c}-0.127 \\
(0.099)\end{array}$ & $\begin{array}{l}0.216^{* *} \\
(0.087)\end{array}$ & $\begin{array}{l}-0.091 \\
(0.117)\end{array}$ & $\begin{array}{l}-0.083 \\
(0.128)\end{array}$ & $\begin{array}{c}0.366^{* * *} \\
(0.099)\end{array}$ & $\begin{array}{l}0.219^{* *} \\
(0.092)\end{array}$ & $\begin{array}{c}0.022 \\
(0.221)\end{array}$ \\
\hline $\ln ($ State Frag. $\cdot(t-1))$ & $\begin{array}{c}0.518 \\
(0.334)\end{array}$ & $\begin{array}{l}1.277^{* *} \\
(0.515)\end{array}$ & $\begin{array}{c}0.183 \\
(0.350)\end{array}$ & & & & $\begin{array}{c}0.409 \\
(0.433)\end{array}$ & $\begin{array}{c}0.794 \\
(0.486)\end{array}$ & $\begin{array}{c}-0.572 \\
(0.441)\end{array}$ \\
\hline $\ln \left(\right.$ Effectiveness $\left._{(t-1)}\right)$ & & & & $\begin{array}{l}-0.136 \\
(0.457)\end{array}$ & $\begin{array}{c}1.618^{* * *} \\
(0.584)\end{array}$ & $\begin{array}{l}-0.298 \\
(0.588)\end{array}$ & & & \\
\hline $\ln \left(\operatorname{Legitimacy}_{(t-1)}\right)$ & & & & $\begin{array}{c}1.245^{* * *} \\
(0.288)\end{array}$ & $\begin{array}{c}0.479 \\
(0.352)\end{array}$ & $\begin{array}{c}0.957^{* *} \\
(0.468)\end{array}$ & & & \\
\hline $\ln$ (Ruggedness) & & & & & & & $\begin{array}{c}0.104 \\
(0.077)\end{array}$ & $\begin{array}{c}0.364^{* * *} \\
(0.061)\end{array}$ & $\begin{array}{l}-0.109 \\
(0.188)\end{array}$ \\
\hline ln(Real PCGDP 1950) & & & & & & & $\begin{array}{c}1.436^{* * *} \\
(0.269)\end{array}$ & $\begin{array}{c}1.511^{* * *} \\
(0.273)\end{array}$ & $\begin{array}{c}0.413 \\
(0.544)\end{array}$ \\
\hline $\ln$ (Dist. Coast) & & & & & & & $\begin{array}{c}0.713^{* * *} \\
(0.160)\end{array}$ & $\begin{array}{c}1.683^{* * *} \\
(0.228)\end{array}$ & $\begin{array}{l}1.458^{* * *} \\
(0.187)\end{array}$ \\
\hline $\ln$ (Dist. Att. Slave) & & & & & & & $\begin{array}{l}0.683^{* *} \\
(0.315)\end{array}$ & $\begin{array}{l}-0.555 \\
(0.659)\end{array}$ & $\begin{array}{c}0.123 \\
(0.348)\end{array}$ \\
\hline $\ln$ (Dist. Indian Slave) & & & & & & & $\begin{array}{l}0.339^{*} \\
(0.200)\end{array}$ & $\begin{array}{c}-0.778^{* * *} \\
(0.208)\end{array}$ & $\begin{array}{c}0.474 \\
(0.535)\end{array}$ \\
\hline British Colony & & & & & & & $\begin{array}{c}0.748 \\
(0.530)\end{array}$ & $\begin{array}{l}-1.201^{*} \\
(0.644)\end{array}$ & $\begin{array}{c}0.226 \\
(0.791)\end{array}$ \\
\hline France Colony & & & & & & & $\begin{array}{c}0.468 \\
(0.389)\end{array}$ & $\begin{array}{l}-0.343 \\
(0.554)\end{array}$ & $\begin{array}{l}-0.240 \\
(0.878)\end{array}$ \\
\hline Portugal Colony & & & & & & & $\begin{array}{c}0.782 \\
(0.628)\end{array}$ & $\begin{array}{c}0.000 \\
(.)\end{array}$ & $\begin{array}{c}1.333 \\
(1.453)\end{array}$ \\
\hline lnalpha & $\begin{array}{c}0.467^{* * *} \\
(0.058)\end{array}$ & $\begin{array}{c}0.239^{* * *} \\
(0.085)\end{array}$ & $\begin{array}{c}0.300^{* * *} \\
(0.082)\end{array}$ & $\begin{array}{c}0.647^{* * *} \\
(0.051)\end{array}$ & $\begin{array}{c}0.484^{* * *} \\
(0.091)\end{array}$ & $\begin{array}{c}0.547^{* * *} \\
(0.072)\end{array}$ & $\begin{array}{c}0.499^{* * *} \\
(0.063)\end{array}$ & $\begin{array}{c}0.118 \\
(0.105)\end{array}$ & $\begin{array}{c}0.350^{* * * *} \\
(0.082)\end{array}$ \\
\hline LogLik & -2277.981 & -941.786 & -1276.286 & -2355.352 & -999.388 & -1314.813 & -2175.525 & -923.735 & -1178.669 \\
\hline Pseudo-R2 & 0.083 & 0.122 & 0.062 & 0.047 & 0.060 & 0.034 & 0.084 & 0.139 & 0.061 \\
\hline Obs & 484 & 253 & 231 & 471 & 240 & 231 & 467 & 253 & 214 \\
\hline
\end{tabular}


Table 4: Negative Binomial: Government/Related Parties (Secondary Education)

\begin{tabular}{|c|c|c|c|c|c|c|c|c|c|}
\hline \multirow[b]{2}{*}{ (1) } & \multicolumn{3}{|c|}{ Globalisation } & \multicolumn{3}{|c|}{ Fragility } & \multicolumn{3}{|c|}{ Geographic } \\
\hline & $\begin{array}{l}(2) \\
\text { All }\end{array}$ & $\begin{array}{l}(3) \\
\text { LQ }\end{array}$ & $\begin{array}{l}(4) \\
\text { HQ }\end{array}$ & $\begin{array}{l}\text { (5) } \\
\text { All }\end{array}$ & $\begin{array}{l}(6) \\
\text { LQ }\end{array}$ & $\begin{array}{l}\text { (7) } \\
\text { HQ }\end{array}$ & $\begin{array}{l}(8) \\
\text { All }\end{array}$ & $\begin{array}{l}(9) \\
\text { LQ }\end{array}$ & $\begin{array}{l}(10) \\
\mathrm{HQ}\end{array}$ \\
\hline $\ln ($ Military Exp.t-1) & $\begin{array}{l}0.780^{* * *} \\
(0.144)\end{array}$ & $\begin{array}{c}0.146 \\
(0.262)\end{array}$ & $\begin{array}{l}0.516^{* * *} \\
(0.180)\end{array}$ & $\begin{array}{l}0.624^{* * *} \\
(0.168)\end{array}$ & $\begin{array}{c}0.745^{* * *} \\
(0.258)\end{array}$ & $\begin{array}{c}0.346 \\
(0.226)\end{array}$ & $\begin{array}{l}0.332^{*} \\
(0.188)\end{array}$ & $\begin{array}{l}-0.671^{* *} \\
(0.277)\end{array}$ & $\begin{array}{l}-0.219 \\
(0.340)\end{array}$ \\
\hline $\ln \left(\right.$ Real $\left.\operatorname{PCGDP}_{(t-1)}\right)$ & $\begin{array}{l}0.582^{* * *} \\
(0.196)\end{array}$ & $\begin{array}{c}-0.166 \\
(0.265)\end{array}$ & $\begin{array}{c}0.763^{* * *} \\
(0.232)\end{array}$ & $\begin{array}{c}0.149 \\
(0.236)\end{array}$ & $\begin{array}{l}-0.205 \\
(0.313)\end{array}$ & $\begin{array}{l}-0.204 \\
(0.226)\end{array}$ & $\begin{array}{l}-0.238 \\
(0.330)\end{array}$ & $\begin{array}{c}-1.675^{* * *} \\
(0.355)\end{array}$ & $\begin{array}{c}-0.001 \\
(0.546)\end{array}$ \\
\hline $\ln \left(\right.$ Globalisation $\left._{(t-1)}\right)$ & & & & $\begin{array}{c}1.009 \\
(0.798)\end{array}$ & $\begin{array}{l}-1.678 \\
(1.185)\end{array}$ & $\begin{array}{l}1.675^{*} \\
(0.959)\end{array}$ & $\begin{array}{l}0.545 \\
(1.027)\end{array}$ & $\begin{array}{c}3.652^{* * *} \\
(1.167)\end{array}$ & $\begin{array}{l}-1.232 \\
(1.317)\end{array}$ \\
\hline $\ln ($ Econ. Globalisation $(t-1))$ & $\begin{array}{c}-0.941^{* *} \\
(0.479)\end{array}$ & $\begin{array}{c}-3.191^{* * *} \\
(1.064)\end{array}$ & $\begin{array}{l}-1.755^{* * *} \\
(0.539)\end{array}$ & & & & & & \\
\hline $\ln ($ Social Globalisation $(t-1))$ & $\begin{array}{c}-2.493^{* * *} \\
(0.530)\end{array}$ & $\begin{array}{l}-0.386 \\
(0.786)\end{array}$ & $\begin{array}{c}-1.479^{* *} \\
(0.723)\end{array}$ & & & & & & \\
\hline $\ln$ (Pol. Globalisation) & $\begin{array}{c}2.751^{* * *} \\
(0.396)\end{array}$ & $\begin{array}{c}2.850^{* * *} \\
(0.672)\end{array}$ & $\begin{array}{c}3.092^{* * *} \\
(0.508)\end{array}$ & & & & & & \\
\hline $\ln \left(\right.$ Resource Rents $\left._{(t-1)}\right)$ & $\begin{array}{c}0.364^{* * *} \\
(0.113)\end{array}$ & $\begin{array}{c}0.532^{* * *} \\
(0.195)\end{array}$ & $\begin{array}{l}0.533^{* * *} \\
(0.140)\end{array}$ & $\begin{array}{c}0.709^{* * *} \\
(0.137)\end{array}$ & $\begin{array}{c}0.622^{* * *} \\
(0.163)\end{array}$ & $\begin{array}{c}0.960^{* * *} \\
(0.142)\end{array}$ & $\begin{array}{c}0.678^{* * *} \\
(0.130)\end{array}$ & $\begin{array}{c}0.380^{* * *} \\
(0.142)\end{array}$ & $\begin{array}{c}0.843^{* * *} \\
(0.283)\end{array}$ \\
\hline $\ln \left(\right.$ Secondary Educ. $\left.{ }_{(t-1)}\right)$ & $\begin{array}{c}0.713^{* * *} \\
(0.234)\end{array}$ & $\begin{array}{l}1.035^{* *} \\
(0.404)\end{array}$ & $\begin{array}{l}-0.088 \\
(0.385)\end{array}$ & $\begin{array}{l}0.645^{* *} \\
(0.301)\end{array}$ & $\begin{array}{c}0.900^{* * *} \\
(0.344)\end{array}$ & $\begin{array}{c}0.426 \\
(0.288)\end{array}$ & $\begin{array}{c}0.338 \\
(0.344)\end{array}$ & $\begin{array}{l}0.708^{* *} \\
(0.347)\end{array}$ & $\begin{array}{l}1.183^{* *} \\
(0.576)\end{array}$ \\
\hline Ln(Pop. Density $(t-1))$ & $\begin{array}{l}0.197^{*} \\
(0.104)\end{array}$ & $\begin{array}{c}-0.610^{* * * *} \\
(0.170)\end{array}$ & $\begin{array}{c}0.016 \\
(0.182)\end{array}$ & $\begin{array}{l}0.181^{*} \\
(0.109)\end{array}$ & $\begin{array}{l}-0.129 \\
(0.161)\end{array}$ & $\begin{array}{c}-0.086 \\
(0.146)\end{array}$ & $\begin{array}{l}0.224^{*} \\
(0.125)\end{array}$ & $\begin{array}{c}-0.400^{* *} \\
(0.166)\end{array}$ & $\begin{array}{c}0.085 \\
(0.244)\end{array}$ \\
\hline $\ln ($ State Frag. $\cdot(t-1))$ & $\begin{array}{l}0.701^{*} \\
(0.380)\end{array}$ & $\begin{array}{l}-0.843 \\
(0.843)\end{array}$ & $\begin{array}{c}0.553 \\
(0.390)\end{array}$ & & & & $\begin{array}{l}1.110^{* *} \\
(0.453)\end{array}$ & $\begin{array}{l}-1.111^{*} \\
(0.596)\end{array}$ & $\begin{array}{l}-0.225 \\
(0.532)\end{array}$ \\
\hline $\ln \left(\right.$ Effectiveness $\left._{(t-1)}\right)$ & & & & $\begin{array}{l}0.409 \\
(0.505)\end{array}$ & $\begin{array}{l}-1.141 \\
(0.856)\end{array}$ & $\begin{array}{l}-1.114 \\
(0.691)\end{array}$ & & & \\
\hline $\ln \left(\right.$ Legitimacy $\left._{(t-1)}\right)$ & & & & $\begin{array}{c}1.154^{* * *} \\
(0.310)\end{array}$ & $\begin{array}{c}0.275 \\
(0.365)\end{array}$ & $\begin{array}{c}1.937^{* * *} \\
(0.465)\end{array}$ & & & \\
\hline $\ln$ (Ruggedness) & & & & & & & $\begin{array}{l}0.164^{* *} \\
(0.081)\end{array}$ & $\begin{array}{c}0.399 * * * \\
(0.073)\end{array}$ & $\begin{array}{c}-0.314^{*} \\
(0.164)\end{array}$ \\
\hline $\ln ($ Real PCGDP 1950) & & & & & & & $\begin{array}{l}1.421^{* * *} \\
(0.326)\end{array}$ & $\begin{array}{l}2.400^{* * *} \\
(0.352)\end{array}$ & $\begin{array}{l}1.125 \\
(0.781)\end{array}$ \\
\hline $\ln$ (Dist. Coast) & & & & & & & $\begin{array}{l}0.416^{* *} \\
(0.188)\end{array}$ & $\begin{array}{c}2.175 * * * \\
(0.316)\end{array}$ & $\begin{array}{l}1.458^{* * *} \\
(0.197)\end{array}$ \\
\hline $\ln$ (Dist. Atl. Slave) & & & & & & & $\begin{array}{l}1.203^{* * *} \\
(0.389)\end{array}$ & $\begin{array}{l}3.438^{* * *} \\
(0.810)\end{array}$ & $\begin{array}{c}0.518 \\
(0.642)\end{array}$ \\
\hline $\ln$ (Dist. Indian Slave) & & & & & & & $\begin{array}{l}0.668^{* *} \\
(0.270)\end{array}$ & $\begin{array}{l}0.583^{*} \\
(0.348)\end{array}$ & $\begin{array}{l}-1.262^{*} \\
(0.746)\end{array}$ \\
\hline British Colony & & & & & & & $\begin{array}{c}0.210 \\
(0.604)\end{array}$ & $\begin{array}{l}0.998^{*} \\
(0.511)\end{array}$ & $\begin{array}{c}0.685 \\
(0.898)\end{array}$ \\
\hline France Colony & & & & & & & $\begin{array}{l}-0.190 \\
(0.454)\end{array}$ & $\begin{array}{l}2.614^{* * *} \\
(0.449)\end{array}$ & $\begin{array}{l}2.891 * * \\
(1.344)\end{array}$ \\
\hline Portugal Colony & & & & & & & $\begin{array}{c}0.564 \\
(0.796)\end{array}$ & $\begin{array}{c}0.000 \\
(.)\end{array}$ & $\begin{array}{l}3.282^{* *} \\
(1.627)\end{array}$ \\
\hline Inalpha & $\begin{array}{c}0.920^{* * *} \\
(0.077)\end{array}$ & $\begin{array}{c}0.789^{* * *} \\
(0.106)\end{array}$ & $\begin{array}{c}0.691^{* * *} \\
(0.100)\end{array}$ & $\begin{array}{c}1.081^{* * *} \\
(0.071)\end{array}$ & $\begin{array}{c}0.904^{* * *} \\
(0.105)\end{array}$ & $\begin{array}{l}0.865^{* * *} \\
(0.091)\end{array}$ & $\begin{array}{c}0.949^{* * *} \\
(0.070)\end{array}$ & $\begin{array}{l}0.310^{* *} \\
(0.123)\end{array}$ & $\begin{array}{c}0.739^{* * *} \\
(0.098) \\
\end{array}$ \\
\hline LogLik & -1528.613 & -611.574 & -876.718 & -1585.263 & $\begin{array}{l}-624.769 \\
\end{array}$ & -916.545 & -1427.272 & -565.312 & -779.978 \\
\hline Pseudo-R2 & 0.084 & 0.105 & 0.067 & 0.049 & 0.063 & 0.046 & 0.085 & 0.173 & 0.070 \\
\hline Obs & 484 & 264 & 220 & 471 & 245 & 226 & 467 & 264 & 203 \\
\hline
\end{tabular}


Table 5: Negative Binomial: Groups/Militias (Secondary Education)

\begin{tabular}{|c|c|c|c|c|c|c|c|c|c|}
\hline \multirow[b]{2}{*}{ (1) } & \multicolumn{3}{|c|}{ Globalisation } & \multicolumn{3}{|c|}{ Fragility } & \multicolumn{3}{|c|}{ Geographic } \\
\hline & $\begin{array}{l}\text { (2) } \\
\text { All }\end{array}$ & $\begin{array}{l}(3) \\
\text { LQ }\end{array}$ & $\begin{array}{l}\text { (4) } \\
\text { HQ }\end{array}$ & $\begin{array}{l}\text { (5) } \\
\text { All }\end{array}$ & $\begin{array}{l}(6) \\
\text { LQ }\end{array}$ & $\begin{array}{l}\text { (7) } \\
\text { HQ }\end{array}$ & $\begin{array}{l}\text { (8) } \\
\text { All }\end{array}$ & $\begin{array}{l}\text { (9) } \\
\text { LQ }\end{array}$ & $\begin{array}{l}(10) \\
\text { HQ }\end{array}$ \\
\hline $\ln ($ Military Exp. $t-1)$ & $\begin{array}{c}0.469^{* * *} \\
(0.133)\end{array}$ & $\begin{array}{c}-0.321 \\
(0.204)\end{array}$ & $\begin{array}{c}0.642^{* * *} \\
(0.171)\end{array}$ & $\begin{array}{l}0.333^{* *} \\
(0.150)\end{array}$ & $\begin{array}{l}-0.009 \\
(0.162)\end{array}$ & $\begin{array}{c}0.872^{* * *} \\
(0.224)\end{array}$ & $\begin{array}{l}0.304^{*} \\
(0.177)\end{array}$ & $\begin{array}{c}0.024 \\
(0.305)\end{array}$ & $\begin{array}{c}1.269^{* * *} \\
(0.317)\end{array}$ \\
\hline $\ln \left(\right.$ Real $\left.\operatorname{PCGDP}_{(t-1)}\right)$ & $\begin{array}{c}0.077 \\
(0.151)\end{array}$ & $\begin{array}{c}-0.639^{* *} \\
(0.279)\end{array}$ & $\begin{array}{l}0.331^{*} \\
(0.178)\end{array}$ & $\begin{array}{c}-0.373^{* *} \\
(0.158)\end{array}$ & $\begin{array}{l}-0.450 \\
(0.278)\end{array}$ & $\begin{array}{l}-0.186 \\
(0.218)\end{array}$ & $\begin{array}{c}-1.116^{* * *} \\
(0.344)\end{array}$ & $\begin{array}{c}-0.714^{* *} \\
(0.333)\end{array}$ & $\begin{array}{c}-0.039 \\
(0.478)\end{array}$ \\
\hline $\ln \left(\right.$ Globalisation $\left._{(t-1)}\right)$ & & & & $\begin{array}{l}1.942^{* *} \\
(0.811)\end{array}$ & $\begin{array}{c}2.288 * * * \\
(0.773)\end{array}$ & $\begin{array}{c}3.765^{* * *} \\
(1.025)\end{array}$ & $\begin{array}{c}0.221 \\
(1.111)\end{array}$ & $\begin{array}{l}2.565^{* *} \\
(1.252)\end{array}$ & $\begin{array}{c}0.999 \\
(1.545)\end{array}$ \\
\hline $\ln ($ Econ. Globalisation $(t-1))$ & $\begin{array}{c}-1.264^{* * *} \\
(0.483)\end{array}$ & $\begin{array}{c}2.855^{* * *} \\
(0.671)\end{array}$ & $\begin{array}{c}-1.530^{* * *} \\
(0.536)\end{array}$ & & & & & & \\
\hline $\ln ($ Social Globalisation $(t-1)$ & $\begin{array}{c}-1.167^{* *} \\
(0.457)\end{array}$ & $\begin{array}{c}-4.143^{* * *} \\
(0.653)\end{array}$ & $\begin{array}{l}-0.722 \\
(0.632)\end{array}$ & & & & & & \\
\hline $\ln ($ Pol. Globalisation $)$ & $\begin{array}{c}3.350^{* * *} \\
(0.327)\end{array}$ & $\begin{array}{c}4.996 \text { **** } \\
(0.792)\end{array}$ & $\begin{array}{c}3.838^{* * *} \\
(0.377)\end{array}$ & & & & & & \\
\hline $\ln \left(\operatorname{Resource} \operatorname{Rents}_{(t-1)}\right)$ & $\begin{array}{c}0.260^{* *} \\
(0.115)\end{array}$ & $\begin{array}{c}0.096 \\
(0.134)\end{array}$ & $\begin{array}{l}0.410^{* *} \\
(0.167)\end{array}$ & $\begin{array}{c}0.403^{* * *} \\
(0.116)\end{array}$ & $\begin{array}{c}0.521^{* * *} \\
(0.118)\end{array}$ & $\begin{array}{c}0.690^{* * *} \\
(0.176)\end{array}$ & $\begin{array}{c}0.532^{* * *} \\
(0.145)\end{array}$ & $\begin{array}{c}0.327^{* * *} \\
(0.108)\end{array}$ & $\begin{array}{c}0.167 \\
(0.218)\end{array}$ \\
\hline $\ln ($ Secondary Educ. $(t-1))$ & $\begin{array}{c}0.880^{* * *} \\
(0.268)\end{array}$ & $\begin{array}{c}2.489^{* * *} \\
(0.361)\end{array}$ & $\begin{array}{c}0.193 \\
(0.346)\end{array}$ & $\begin{array}{c}1.203^{* * *} \\
(0.209)\end{array}$ & $\begin{array}{c}1.541^{* * *} \\
(0.276)\end{array}$ & $\begin{array}{c}0.551^{*} \\
(0.281)\end{array}$ & $\begin{array}{c}1.254^{* * *} \\
(0.311)\end{array}$ & $\begin{array}{c}1.612^{* * *} \\
(0.327)\end{array}$ & $\begin{array}{l}0.915^{*} \\
(0.496)\end{array}$ \\
\hline Ln(Pop. Density $(t-1))$ & $\begin{array}{c}0.074 \\
(0.086)\end{array}$ & $\begin{array}{c}-0.795^{* * *} \\
(0.144)\end{array}$ & $\begin{array}{c}0.025 \\
(0.131)\end{array}$ & $\begin{array}{l}0.189^{* *} \\
(0.090)\end{array}$ & $\begin{array}{c}-0.419^{* * *} \\
(0.135)\end{array}$ & $\begin{array}{c}0.056 \\
(0.145)\end{array}$ & $\begin{array}{c}0.426^{* * *} \\
(0.132)\end{array}$ & $\begin{array}{c}0.330^{* * *} \\
(0.124)\end{array}$ & $\begin{array}{c}0.291 \\
(0.232)\end{array}$ \\
\hline $\ln ($ State Frag. $\cdot(t-1))$ & $\begin{array}{c}1.610^{* * *} \\
(0.353)\end{array}$ & $\begin{array}{c}1.371^{* * *} \\
(0.492)\end{array}$ & $\begin{array}{c}1.536^{* * *} \\
(0.416)\end{array}$ & & & & $\begin{array}{l}1.081^{* *} \\
(0.481)\end{array}$ & $\begin{array}{c}2.261^{* * *} \\
(0.571)\end{array}$ & $\begin{array}{l}-1.341^{*} \\
(0.769)\end{array}$ \\
\hline $\ln \left(\right.$ Effectiveness $\left._{(t-1)}\right)$ & & & & $\begin{array}{c}0.456 \\
(0.466)\end{array}$ & $\begin{array}{l}1.561^{* *} \\
(0.667)\end{array}$ & $\begin{array}{c}0.440 \\
(0.577)\end{array}$ & & & \\
\hline $\ln \left(\right.$ Legitimacy $\left._{(t-1)}\right)$ & & & & $\begin{array}{c}1.996 * * * \\
(0.256)\end{array}$ & $\begin{array}{c}1.014^{* * *} \\
(0.270)\end{array}$ & $\begin{array}{c}2.092^{* * *} \\
(0.430)\end{array}$ & & & \\
\hline ln(Ruggedness) & & & & & & & $\begin{array}{c}0.116 \\
(0.109)\end{array}$ & $\begin{array}{c}0.595^{* * *} \\
(0.077)\end{array}$ & $\begin{array}{c}-0.692^{* * *} \\
(0.229)\end{array}$ \\
\hline $\ln ($ Real PCGDP 1950) & & & & & & & $\begin{array}{c}1.600^{* * *} \\
(0.352)\end{array}$ & $\begin{array}{c}1.221^{* * *} \\
(0.443)\end{array}$ & $\begin{array}{l}-0.060 \\
(0.625)\end{array}$ \\
\hline $\ln$ (Dist. Coast) & & & & & & & $\begin{array}{c}0.913^{* * *} \\
(0.217)\end{array}$ & $\begin{array}{c}2.261^{* * *} \\
(0.347)\end{array}$ & $\begin{array}{c}2.643^{* * *} \\
(0.278)\end{array}$ \\
\hline $\ln$ (Dist. Atl. Slave) & & & & & & & $\begin{array}{c}0.019 \\
(0.364)\end{array}$ & $\begin{array}{c}-2.036^{* * *} \\
(0.642)\end{array}$ & $\begin{array}{c}-1.092^{* *} \\
(0.478)\end{array}$ \\
\hline $\ln$ (Dist. Indian Slave) & & & & & & & $\begin{array}{c}0.413 \\
(0.282)\end{array}$ & $\begin{array}{c}-1.469^{* * *} \\
(0.283)\end{array}$ & $\begin{array}{c}0.137 \\
(0.500)\end{array}$ \\
\hline British Colony & & & & & & & $\begin{array}{l}1.721^{* *} \\
(0.700)\end{array}$ & $\begin{array}{l}-0.310 \\
(0.528)\end{array}$ & $\begin{array}{c}0.704 \\
(0.747)\end{array}$ \\
\hline France Colony & & & & & & & $\begin{array}{l}0.885^{*} \\
(0.485)\end{array}$ & $\begin{array}{l}-0.595 \\
(0.423)\end{array}$ & $\begin{array}{l}-0.313 \\
(0.897)\end{array}$ \\
\hline Portugal Colony & & & & & & & $\begin{array}{l}1.053^{*} \\
(0.614) \\
\end{array}$ & $\begin{array}{c}0.000 \\
(.)\end{array}$ & $\begin{array}{c}0.756 \\
(1.626) \\
\end{array}$ \\
\hline lnalpha & $\begin{array}{c}0.835^{* * *} \\
(0.068)\end{array}$ & $\begin{array}{c}0.469^{* * *} \\
(0.123)\end{array}$ & $\begin{array}{c}0.731^{* * *} \\
(0.078)\end{array}$ & $\begin{array}{c}0.970^{* * *} \\
(0.060)\end{array}$ & $\begin{array}{c}0.740^{* * *} \\
(0.116)\end{array}$ & $\begin{array}{c}0.910^{* * *} \\
(0.072)\end{array}$ & $\begin{array}{c}0.855^{* * *} \\
(0.069)\end{array}$ & $\begin{array}{c}0.061 \\
(0.134)\end{array}$ & $\begin{array}{c}0.636^{* * *} \\
(0.099)\end{array}$ \\
\hline LogLik & -1758.627 & -599.857 & -1111.284 & -1805.052 & -635.138 & -1138.509 & -1679.113 & -566.572 & -1018.612 \\
\hline Pseudo-R2 & 0.082 & 0.143 & 0.058 & 0.053 & 0.084 & 0.034 & 0.085 & 0.190 & 0.073 \\
\hline Obs & 484 & 230 & 254 & 471 & 217 & 254 & 467 & 230 & 237 \\
\hline
\end{tabular}


Table 6: Negative Binomial: Civilians/Protests (Secondary Education)

\begin{tabular}{|c|c|c|c|c|c|c|c|c|c|}
\hline \multirow[b]{2}{*}{ (1) } & \multicolumn{3}{|c|}{ Globalisation } & \multicolumn{3}{|c|}{ Fragility } & \multicolumn{3}{|c|}{ Geographic } \\
\hline & $\begin{array}{l}(2) \\
\text { All }\end{array}$ & $\begin{array}{l}(3) \\
\text { LQ }\end{array}$ & $\begin{array}{l}(4) \\
\mathrm{HQ}\end{array}$ & $\begin{array}{l}(5) \\
\text { All }\end{array}$ & $\begin{array}{l}(6) \\
\text { LQ }\end{array}$ & $\begin{array}{l}(7) \\
\mathrm{HQ}\end{array}$ & $\begin{array}{l}(8) \\
\text { All }\end{array}$ & $\begin{array}{l}(9) \\
\text { LQ }\end{array}$ & $\begin{array}{l}(10) \\
\text { HQ }\end{array}$ \\
\hline $\ln ($ Military Exp. $t-1)$ & $\begin{array}{c}0.195 \\
(0.131)\end{array}$ & $\begin{array}{l}1.011^{* *} \\
(0.439)\end{array}$ & $\begin{array}{l}-0.018 \\
(0.169)\end{array}$ & $\begin{array}{c}0.154 \\
(0.187)\end{array}$ & $\begin{array}{l}0.587^{* *} \\
(0.231)\end{array}$ & $\begin{array}{l}0.149 \\
(0.274)\end{array}$ & $\begin{array}{c}0.202 \\
(0.179)\end{array}$ & $\begin{array}{c}0.525 \\
(0.384)\end{array}$ & $\begin{array}{c}0.144 \\
(0.272)\end{array}$ \\
\hline $\ln \left(\right.$ Real $\left.\operatorname{PCGDP}_{(t-1)}\right)$ & $\begin{array}{l}-0.037 \\
(0.137)\end{array}$ & $\begin{array}{c}-1.217^{* * *} \\
(0.267)\end{array}$ & $\begin{array}{c}0.211 \\
(0.145)\end{array}$ & $\begin{array}{c}-0.531^{* * *} \\
(0.168)\end{array}$ & $\begin{array}{c}-1.140^{* * * *} \\
(0.274)\end{array}$ & $\begin{array}{c}-0.499^{* *} \\
(0.235)\end{array}$ & $\begin{array}{c}-0.846^{* * *} \\
(0.195)\end{array}$ & $\begin{array}{c}-1.450^{* * *} \\
(0.395)\end{array}$ & $\begin{array}{c}0.159 \\
(0.215)\end{array}$ \\
\hline $\ln \left(\right.$ Globalisation $\left._{(t-1)}\right)$ & & & & $\begin{array}{c}4.917^{* * *} \\
(0.828)\end{array}$ & $\begin{array}{c}5.267^{* * *} \\
(1.201)\end{array}$ & $\begin{array}{c}3.844^{* * *} \\
(0.867)\end{array}$ & $\begin{array}{c}4.817^{* * *} \\
(0.831)\end{array}$ & $\begin{array}{l}4.016^{* *} \\
(1.800)\end{array}$ & $\begin{array}{l}2.552^{* *} \\
(1.073)\end{array}$ \\
\hline $\ln ($ Econ. Globalisation $(t-1))$ & $\begin{array}{l}-0.620 \\
(0.469)\end{array}$ & $\begin{array}{c}0.977 \\
(1.096)\end{array}$ & $\begin{array}{c}-1.820^{* * *} \\
(0.489)\end{array}$ & & & & & & \\
\hline $\ln \left(\right.$ Social Globalisation $\left._{(t-1)}\right)$ & $\begin{array}{c}1.310^{* * *} \\
(0.357)\end{array}$ & $\begin{array}{c}0.191 \\
(0.697)\end{array}$ & $\begin{array}{l}1.072^{* *} \\
(0.440)\end{array}$ & & & & & & \\
\hline $\ln$ (Pol. Globalisation) & $\begin{array}{l}3.694^{* * *} \\
(0.306)\end{array}$ & $\begin{array}{l}3.919^{* * *} \\
(0.825)\end{array}$ & $\begin{array}{l}3.053^{* * *} \\
(0.305)\end{array}$ & & & & & & \\
\hline $\ln ($ Resource Rents $(t-1))$ & $\begin{array}{c}0.717^{* * *} \\
(0.080)\end{array}$ & $\begin{array}{c}0.413^{* * *} \\
(0.145)\end{array}$ & $\begin{array}{l}0.650^{* * *} \\
(0.107)\end{array}$ & $\begin{array}{c}0.676^{* * *} \\
(0.098)\end{array}$ & $\begin{array}{c}0.638^{* * *} \\
(0.136)\end{array}$ & $\begin{array}{c}0.618^{* * *} \\
(0.121)\end{array}$ & $\begin{array}{l}0.876^{* * *} \\
(0.099)\end{array}$ & $\begin{array}{c}0.702^{* * *} \\
(0.172)\end{array}$ & $\begin{array}{l}0.594^{* * *} \\
(0.147)\end{array}$ \\
\hline $\ln \left(\right.$ Secondary Educ. $\left.{ }_{(t-1)}\right)$ & $\begin{array}{c}0.604^{* * *} \\
(0.208)\end{array}$ & $\begin{array}{c}1.076^{* * *} \\
(0.275)\end{array}$ & $\begin{array}{c}1.058^{* * *} \\
(0.303)\end{array}$ & $\begin{array}{c}1.046^{* * *} \\
(0.232)\end{array}$ & $\begin{array}{c}1.306^{* * *} \\
(0.279)\end{array}$ & $\begin{array}{c}1.424^{* * *} \\
(0.370)\end{array}$ & $\begin{array}{c}0.964^{* * *} \\
(0.272)\end{array}$ & $\begin{array}{c}1.756^{* * *} \\
(0.466)\end{array}$ & $\begin{array}{c}1.917^{* * *} \\
(0.429)\end{array}$ \\
\hline $\operatorname{Ln}($ Pop. Density $(t-1))$ & $\begin{array}{c}0.117 \\
(0.072)\end{array}$ & $\begin{array}{l}-0.122 \\
(0.136)\end{array}$ & $\begin{array}{l}-0.150 \\
(0.140)\end{array}$ & $\begin{array}{l}0.325^{* * *} \\
(0.086)\end{array}$ & $\begin{array}{l}-0.013 \\
(0.135)\end{array}$ & $\begin{array}{c}0.126 \\
(0.213)\end{array}$ & $\begin{array}{l}0.363^{* * *} \\
(0.093)\end{array}$ & $\begin{array}{l}-0.133 \\
(0.143)\end{array}$ & $\begin{array}{l}0.207 \\
(0.261)\end{array}$ \\
\hline $\ln ($ State Frag. $\cdot(t-1))$ & $\begin{array}{l}-0.010 \\
(0.331)\end{array}$ & $\begin{array}{l}-0.055 \\
(0.754)\end{array}$ & $\begin{array}{l}-0.124 \\
(0.351)\end{array}$ & & & & $\begin{array}{l}-0.409 \\
(0.433)\end{array}$ & $\begin{array}{l}1.422^{*} \\
(0.848)\end{array}$ & $\begin{array}{l}-0.230 \\
(0.474)\end{array}$ \\
\hline $\ln \left(\right.$ Effectiveness $\left._{(t-1)}\right)$ & & & & $\begin{array}{c}-0.574 \\
(0.469)\end{array}$ & $\begin{array}{c}0.744 \\
(0.632)\end{array}$ & $\begin{array}{l}-1.429 * * * \\
(0.541)\end{array}$ & & & \\
\hline $\ln \left(\operatorname{Legitimacy}_{(t-1)}\right)$ & & & & $\begin{array}{l}0.604^{*} \\
(0.333)\end{array}$ & $\begin{array}{c}0.193 \\
(0.359)\end{array}$ & $\begin{array}{l}1.210^{* * *} \\
(0.400)\end{array}$ & & & \\
\hline $\ln$ (Ruggedness) & & & & & & & $\begin{array}{c}0.051 \\
(0.067)\end{array}$ & $\begin{array}{c}0.049 \\
(0.096)\end{array}$ & $\begin{array}{l}-0.238 * * \\
(0.117)\end{array}$ \\
\hline ln(Real PCGDP 1950) & & & & & & & $\begin{array}{c}1.125^{* * *} \\
(0.215)\end{array}$ & $\begin{array}{c}0.756^{* * *} \\
(0.236)\end{array}$ & $\begin{array}{c}0.344 \\
(0.336)\end{array}$ \\
\hline $\ln$ (Dist. Coast) & & & & & & & $\begin{array}{c}0.504^{* * * *} \\
(0.150)\end{array}$ & $\begin{array}{c}0.138 \\
(0.251)\end{array}$ & $\begin{array}{c}0.820^{* * *} \\
(0.165)\end{array}$ \\
\hline $\ln$ (Dist. Atl. Slave) & & & & & & & $\begin{array}{l}0.764^{* * *} \\
(0.291)\end{array}$ & $\begin{array}{l}1.580^{* *} \\
(0.770)\end{array}$ & $\begin{array}{c}0.282 \\
(0.361)\end{array}$ \\
\hline $\ln$ (Dist. Indian Slave) & & & & & & & $\begin{array}{c}0.138 \\
(0.152)\end{array}$ & $\begin{array}{l}-0.005 \\
(0.378)\end{array}$ & $\begin{array}{l}-0.542^{*} \\
(0.292)\end{array}$ \\
\hline British Colony & & & & & & & $\begin{array}{l}0.869^{* *} \\
(0.443)\end{array}$ & $\begin{array}{l}1.410 \\
(1.050)\end{array}$ & $\begin{array}{l}-0.306 \\
(0.509)\end{array}$ \\
\hline France Colony & & & & & & & $\begin{array}{l}0.864^{* *} \\
(0.385)\end{array}$ & $\begin{array}{c}0.495 \\
(0.596)\end{array}$ & $\begin{array}{c}0.842 \\
(0.587)\end{array}$ \\
\hline Portugal Colony & & & & & & & $\begin{array}{c}0.517 \\
(0.511)\end{array}$ & $\begin{array}{c}0.524 \\
(0.809)\end{array}$ & $\begin{array}{c}0.520 \\
(0.908)\end{array}$ \\
\hline lnalpha & $\begin{array}{c}0.599^{* * *} \\
(0.067)\end{array}$ & $\begin{array}{l}0.350^{* * *} \\
(0.126)\end{array}$ & $\begin{array}{l}0.487^{* * *} \\
(0.089)\end{array}$ & $\begin{array}{c}0.761^{* * *} \\
(0.073)\end{array}$ & $\begin{array}{l}0.462^{* * *} \\
(0.125)\end{array}$ & $\begin{array}{c}0.700^{* * *} \\
(0.099)\end{array}$ & $\begin{array}{c}0.580^{* * *} \\
(0.072)\end{array}$ & $\begin{array}{c}0.126 \\
(0.140)\end{array}$ & $\begin{array}{c}0.508^{* * *} \\
(0.094)\end{array}$ \\
\hline LogLik & -1670.643 & & -1109.210 & -1716.522 & -538.365 & -1142.445 & -1601.437 & -501.884 & -1053.300 \\
\hline Pseudo-R2 & 0.112 & 0.147 & 0.106 & 0.082 & 0.101 & 0.079 & 0.117 & 0.176 & 0.101 \\
\hline Obs & 484 & 200 & 284 & 471 & 187 & 284 & 467 & 200 & 267 \\
\hline
\end{tabular}

